Mon. Not. R. Astron. Soc. 000,1 126()$\quad$ Printed 30 October $2018 \quad$ (MN LATEX style file v2.2)

\title{
Detection of dark galaxies and circum-galactic filaments fluorescently illuminated by a quasar at $\mathrm{z}=2.4^{\star}$
}

\author{
Sebastiano Cantalupo ${ }^{1,2} \dagger$, Simon J. Lilly ${ }^{3}$ and Martin G. Haehnelt ${ }^{1,4}$ \\ ${ }^{1}$ Kavli Institute for Cosmology, Cambridge $\&$ Institute of Astronomy, Madingley Road, Cambridge CB3 OHA, UK. \\ 2 Department of Astronomy and Astrophysics \& UCO/Lick Observatory, University of California, Santa Cruz, CA 95064, USA. \\ 3 Institute for Astronomy, ETH Zurich, Wolfgang-Pauli-Strasse 27, CH-8093 Zurich, Switzerland. \\ ${ }^{4}$ Kavli Institute for Theoretical Physics, University of California, Santa Barbara, CA 93106, USA.
}

Accepted 2012 June 15. Received 2012 June 10; in original form 2012 April 24

\begin{abstract}
A deep narrow-band survey for Ly $\alpha$ emission carried out on the VLT-FORS has revealed $98 \mathrm{Ly} \alpha$ candidates down to a flux limit of $F_{\mathrm{Ly} \alpha} \sim 4 \times 10^{-18} \mathrm{erg} \mathrm{s}^{-1} \mathrm{~cm}^{-2}$ in a volume of 5500 comoving $\mathrm{Mpc}^{3}$ at $z=2.4$ centered on the hyperluminous quasar HE0109-3518. The properties of the detected sources in terms of their i) equivalent width distribution, ii) luminosity function, and iii) the average luminosity versus projected distance from the quasar, all suggest that a large fraction of these objects have been fluorescently "illuminated" by HE0109-3518. This conclusion is supported by comparison with detailed radiative transfer simulations of the effects of the quasar illumination. We therefore have a unique opportunity to directly detect and image in emission dense gas, independent of any associated star formation activity. 18 objects, a much larger fraction than in "blank-field" Ly $\alpha$ surveys at similar redshifts, have a rest-frame Equivalent Width $\left(\mathrm{EW}_{0}\right)$ larger than $240 \AA$, the expected limit for Ly $\alpha$ emission powered by Population II star formation. 12 sources among these do not have any continuum counterpart in a deep V-band imaging of the same field (reaching to $V(1 \sigma) \sim 30.3 \mathrm{AB})$. For these, a stacking analysis indicates $\mathrm{EW}_{0}>800 \AA(1 \sigma)$, effectively ruling out Ly $\alpha$ powered by internal star formation. These sources are thus the best candidates so far for proto-galactic clouds or "dark" galaxies at high-redshift, whose existence has recently been suggested by several theoretical studies. Assuming they are mostly ionized by the quasar radiation, we estimate that their gas masses would be about $M_{\text {gas }} \sim 10^{9} M_{\odot}$ implying that their star formation efficiencies (SFEs) are less than $10^{-11} \mathrm{yr}^{-1}$, equivalent to a gas consumption time of $100 \mathrm{Gyr}$, several times below the SFE of the most gas-rich dwarf galaxies locally, and two hundred times lower than typical massive star-forming galaxies at $z \sim 2$. We have also discovered extended, filamentary gas, also likely illuminated by the quasar, around some of the brightest continuum-detected sources with $\mathrm{EW}_{0}>240 \AA$. The morphology and luminosity of this extended emission are compatible with the expectations for circumgalactic cold streams, as predicted by recent numerical models, but other origins, including tidal stripping, are also possible.
\end{abstract}

Key words:

intergalactic medium - galaxies:formation - galaxies: star formation - galaxies: dwarf - galaxies: high redshift - quasars

\footnotetext{
* Based on observations collected at the European Organisation for Astronomical Research in the Southern Hemisphere, Chile under program 085.A-0171A.

† E-mail:cantal@ucolick.org
}

\section{INTRODUCTION}

The Intergalactic Medium (IGM) plays a crucial role in the formation and evolution of the structures in the Universe. Galaxies form from the IGM that settles into gravitational potential wells deep enough for the gas to collapse (Rees \& Ostriker 1977, White \& Rees 1978). Radiative cooling dis-

(C) RAS 
sipates thermal energy allowing the gas to concentrate at the center of the halo and to generally form a (dense) disk. Following fragmentation, star formation takes place. Most of the observational studies conducted so far have captured the galaxy formation process from this stage on. Despite their importance, the early phases which may involve potential wells too shallow to efficiently form stars are largely unobserved at present and remain poorly constrained. Key questions about how the IGM is converted into stars at highredshift and how efficient this process is remain unanswered.

Recent theoretical studies have suggested that gas-rich, low-mass haloes $\left(10^{9}-10^{11} \mathrm{M}_{\odot}\right)$ at high redshift may have very low star formation efficiencies, as a consequence of lower metallicities (e.g., Gnedin \& Kravtsov 2010, Krumholz \& Dekel 2011), $\mathrm{H}_{2}$ self-regulation effects (e.g., Kuhlen et al. 2012), or reduced cooling accretion rates due to local sources (e.g., Cantalupo 2010). If their star-formation efficiencies are suppressed, these "dark" galaxies can maintain a reservoir of gas that will be available for later star-formation in more massive systems. This phase of suspended activity may be required in order to simultaneously account for several properties of the current galaxy population, like the observed slopes of the SFR-mass and the Tully-Fisher relation, as has been suggested by Bouché et al. (2010).

The potential importance of this early phase of galaxy formation raises the obvious question of how to discover and study such gas clouds that do not have significant starformation. One possibility is try to detect them in HI absorption against background quasars (see, e.g., Rauch 1998 and Wolfe, Gawiser \& Prochaska 2005 for reviews). For instance, recent studies by Fumagalli, O'Meara \& Prochaska (2011) and Cooke et al. (2011) have revealed high column-density systems that have properties which may be compatible with these proto-galactic clouds, given their metallicity consistent with primordial or Population III stars. Unfortunately, these one-dimensional studies cannot directly discriminate between truly isolated clouds or components of larger galactic reservoirs, either around or within galaxies. Detecting these systems in emission rather than in absorption would provide crucial information about their sizes, morphologies and masses, potentially leading to a direct constraint on their star formation efficiencies.

Direct imaging of $\mathrm{HI}$ in the $21 \mathrm{~cm}$ line is unfortunately restricted to the local Universe (e.g. Giovanelli et al. 2005), given the intrinsic faintness of this atomic transition. Observational attempts to detect "dark" galaxies with this technique in the local Universe have not so far produced positive results (e.g. Gavazzi et al. 2008). A significant fraction of local dwarf galaxies do show, however, a much lower star formation efficiency than is found in more massive spiral galaxies (Geha et al. 2006, Schiminovich et al. 2010). Extending these direct imaging studies to high-redshift, where the cosmic star formation rate peaks, may require however an intrinsically brighter tracer of cosmic hydrogen: i.e. the HI Ly $\alpha$ line.

It has been predicted for many years (e.g., Hogan \& Weymann 1987) that the neutral gas that is responsible for quasar absorption lines at high redshift should produce potentially detectable fluorescent Ly $\alpha$ emission at $1216 \AA$ from the incident ionizing radiation field at $\lambda<912 \AA$. For regions that are optically thick to ionizing radiation and are isotropically illuminated from outside, a thin layer around the outer surface should act as a simple fluorescent mirror, converting up to $60 \%$ of the incident ionizing radiation into Ly $\alpha$ photons (Gould \& Weinberg 1996). Velocity field and geometrical effects can reduce the signal by half (Cantalupo et al. 2005). Unfortunately, the cosmic UV background is rather weak - whether predicted theoretically (e.g., Haardt \& Madau 2012) or estimated observationally using the proximity effect in quasar absorption spectra (e.g., Bajtlik, Duncan \& Ostriker 1988, Rauch et al. 1997, Scott et al., 2002, Bolton et al. 2005, Dall'Aglio et al. 2008, D'Odorico et al. 2008). This makes the detection of fluorescent emission from optically thick clouds in the field, i.e. illuminated by the general cosmic UV background, extremely challenging, and perhaps impossible, even on 8-10 m class telescopes (see, e.g., Rauch et al. 2008). However, local enhancements in the ionizing background can be used to substantially increase the signal (e.g., Haiman \& Rees 2001). For instance, gas in the vicinity of a bright quasar may be exposed to a stronger UV flux compared with to an "average" cloud, and are then expected to be correspondingly brighter in fluorescent Ly $\alpha$ (e.g., Cantalupo et al 2005).

The detection of the line-of-sight proximity effect in quasar spectra provides evidence - in absorption - that bright quasars are indeed "illuminating" and ionizing gas clouds within several comoving Mpc scales, at least along our lineof-sight. Searches for a similar effect along the transverse direction using projected pair of quasars, the so called "transverse proximity-effect", have however produced mixed results so far (e.g., Jakobsen et al. 2003, Hennawi \& Prochaska 2007, Worseck et al. 2007, Goncalves, Steidel \& Pettini 2008, Kirkman \& Tytler 2008), indicating possible emission anisotropies or short ages for the quasar bright phase $(<1$ Myr). Detection of quasar fluorescence - the "counterpart" in emission of the proximity effect - may thus provide direct constraints on angular emission properties and lifetimes of these sources.

\subsection{Previous observational attempts to detect fluorescent emission near quasars}

In the last years, we have seen an increase of our knowledge of the quasar fluorescence process with detailed numerical studies (e.g., Cantalupo et al. 2005, Kollmeier et al. 2010). However, until very recently, dedicated observational surveys have been limited to a few, generally unsuccessful, searches. The first fluorescent survey around a quasar, based on the simple expectations from the slab model of Gould \& Weinberg (1996) was performed by Francis \& BlandHawthorn (2004) using a tunable filter imager on the $4 \mathrm{~m}$ Anglo-Australian Telescope. This produced a null result. However, as demonstrated by Cantalupo et al. 2005 (see also the discussion in Cantalupo et al. 2007), this null result is in fact quite consistent with theoretical expectations from quasar illumination considering: i) a more realistic density distribution than simple slab geometry for the gas, ii) the proximity effect of the quasar itself, which reduces the sizes and number density of optically thick clouds. A few years later, Adelberger et al. (2006) discovered, serendipitously,

1 These effects explained the null detection of fluorescent sources. However, it is still unclear why no line emitting galaxies were 
a double peaked Ly $\alpha$ emitter associated with a Damped Ly $\alpha$ absorbing system in the proximity of a bright quasar. The Ly $\alpha$ profile was consistent with expectations from fluorescence and indicated that the gas was certainly optically thick. However, this objects was also associated with a star forming galaxy that was bright enough in the continuum to entirely power - internally - the observed Ly $\alpha$ emission, given its rest-frame Equivalent Width $\left(\mathrm{EW}_{0}\right)$ of about $75 \AA$, well within the range that can be produced by conventional stellar populations (e.g., Schaerer 2002). A systematic survey for other similar systems in quasar pairs by Hennawi et al. (2006) has until now yielded only one Ly $\alpha$ emitting source (Hennawi et al. 2009). However, these authors concluded that this object is likely intrinsic to the quasar host and similar to the $\operatorname{Ly} \alpha$ fuzz detected around many active galactic nuclei (e.g., Heckman et al. 1991; see also Francis \& McDonnell 2006).

Motivated by our own more sophisticated theoretical modeling, in Cantalupo et al. (2007) we carried out a blind spectroscopic survey around a bright quasar at $z=3.1$ using the VLT/FORS2 instrument in a "multi-slit plus filter" mode (Crampton \& Lilly 1999), using two OIII narrow band filters. This method allowed us to perform a deep spectroscopic survey down to a flux limit of $F_{l y \alpha} \sim 2 \times 10^{-18} \mathrm{erg} \mathrm{s}^{-1}$ $\mathrm{cm}^{-2}$ over a significant but non-contiguous volume around the quasar $\left(\sim 1700\right.$ comoving $\left.\mathrm{Mpc}^{3}\right)$. In good agreement with theoretical expectations, we found 13 line emitters consistent with $\operatorname{Ly} \alpha$, a third of which showed signatures of the double-peaked profiles expected from fluorescence. Only 2 of these 13 objects had a significant $(2 \sigma)$ detection of underlying stellar continuum emission in a 2 hour deep $\mathrm{V}$-band image of the same field. Unfortunately, the depth of the Vband image was not sufficient to rule out the presence of internal "illumination" by star formation as the source of the ionizing radiation. However, other indications, including the constraints on the $\operatorname{Ly} \alpha$ surface brightness as a function of distance from the quasar, suggested that about half of the sample could have been indeed fluorescently illuminated by the quasar.

A blind spectroscopic survey has the advantage of reducing the sky-background and giving immediate confirmation of the line-emitting nature of the source. However, there are also several limitations of this approach, including: i) a reduction in the sampled volume, by a factor $\sim 14$ for our "multi-slit plus filter" configuration, compared to narrowband imaging on the same instrument, ii) an inability to detect extended objects, e.g. filamentary structure, with sizes larger than the slit width (2" in our case), iii) the absence of unambiguous information on the sizes and positions of objects (since one spatial dimension is effectively lost), which are crucial to better constrain the $\mathrm{EW}_{0}$ by comparison with a broad-band image.

In this paper, we present a new survey that is based on deep narrow-band (NB) imaging on VLT-FORS that overcomes these limitations and provides the first large statistical sample of cosmic gas clouds that have been fluorescently illuminated by a quasar.

The layout of the paper is as follows. In Section 2, we

detected in the same field (when at least $6 \mathrm{Ly} \alpha$ emitters were expected). present the survey design, observations, data reduction and Ly $\alpha$ candidate selection. In Section 3 we show the observational results. In Section 4 we demonstrate that many of the objects detected have likely been fluorescently illuminated by the quasar. In particular, we compare the EW distribution and Ly $\alpha$ luminosity function with those from field surveys, and examine the relation between object luminosity and the projected distance from the quasar. In Section [5] we discuss the nature and physical properties of this new population of Ly $\alpha$ sources and the implications of our identification as fluorescent emission. In Section 6 we discuss the uncertainties and limitations of our survey. We summarize and conclude the paper in Section 7 . Through the paper, we use a "standard" $\Lambda$ CDM cosmology with $H_{0}=71 \mathrm{~km} \mathrm{~s}^{-1}$ $\mathrm{Mpc}^{-1}, \Omega_{m}=0.27$ and $\Omega_{\Lambda}=0.73$ from WMAP seven-year release (Komatsu et al. 2011).

\section{THE DATA}

\subsection{Survey Design}

Our survey design has been motivated by two simple but crucial requirements: i) to maximize the fluorescently illuminated volume, by selecting one of the brightest quasars in the sky, ii) to minimize the sky-background by having the narrowest bandwidth filter that contains such a volume. In order to meet these two requirements, it is vital that the systemic redshift of the quasar is known with good accuracy, e.g. from low-ionization lines or narrow forbidden lines such as [OIII]. Unfortunately, not many quasars have such a measure - and our previously observed quasar at $z=3.1$ is not one of these - especially if we also require them to be within an existing NB filter. Although we had already several detections around the $z=3.1$ quasar, a large fraction of these would have been too faint to be re-detected in NB imaging. For the above reasons, we designed a new custom NB filter to take advantage of the new blue sensitive E2V detector on FORS and enabling us to select an ultraluminous quasar at $z=2.4$. Due to the lower surface brightness redshift dimming - a factor of about 2 - and the increased throughput, an imaging survey based on a 20 hour NB image at $z=2.4$ should reach a similar depth to our previous spectroscopic survey at $z=3.1$. As we will show, the much larger volume probed by NB imaging results in a substantially larger sample of Ly $\alpha$ sources - about a hundred - allowing us to perform crucial statistical analyses to confirm the fluorescent nature of these sources, e.g. the distribution of Ly $\alpha$ EW compared with similar surveys that did not target bright quasars. Moreover, we are now able to constrain the sizes and masses of these systems and to detect extended, circumgalactic emission.

\subsection{Observations and data reduction}

Observations were taken during four visitor-mode nights at the VLT 8.2m telescope Antu (UT1) on 2010 September 912 using the FORS instrument in imaging mode with the blue sensitive E2V CCD. A custom-built interference filter (FILT_414_4) with central wavelength $\lambda_{\mathrm{NB}}=414.5 \mathrm{~nm}$ and full-width-half-maximum FWHM $=4 \mathrm{~nm}$ has been used to image the field of HE0109-3518 $\left(z_{\text {sys }}=2.4057 \pm 0.0003\right.$, 
$\left.b_{J}=16.7\right)$, sensitive to HI Ly $\alpha$ over a narrow $(\Delta z=0.033)$ redshift range around the quasar. The quasar systemic redshift is precisely constrained by the wavelength of the detected [OIII] $\lambda 5007$ emission line (Shemmer et al. 2004, Marziani et al. 2009). The quasar is radio-quiet. The field was also observed in the $\mathrm{B}_{\mathrm{HIGH}}$ and $\mathrm{V}_{\mathrm{HIGH}}$ filters. A total of 20 hours integration through the narrow-band filter, 6 hours through the V-band (of which 4.5 hours were used) and 0.9 hours through the B-band filter were obtained. The observation $\log$ is reported in Table 1 . Observations were split into separate exposures of $1200 \mathrm{~s}$ in the narrow-band and 120$300 \mathrm{~s}$ in the broad bands. Individual exposures were shifted and rotated with respect to each other using a semi-random shifting pattern of radius 15" and four different position angles $(0,90,180$ and 270 degrees) in order to facilitate the removal of cosmic rays, CCD cosmetic, ghosts and residual flat-field errors. The first part of the night beginning 2010 September 12 suffered from poor seeing conditions, affecting $18 \mathrm{~V}$-band exposures (for a total of 5400s). These have been discarded before image combination. Therefore, the final $\mathrm{V}$ image corresponds to a combined integration time of 4.5 hours.

The images have been reduced using standard routines within the reduction software IRAF, including bias subtraction, flat fielding, CCD illumination correction and image combination. A combination of twilight sky flats and unregistered science frames has been used to produce flat field images and illumination corrections for each band and position angle. Sky subtraction was performed, image by image, fitting a polynomial surface function of order two in order to compensate for small residuals and possible sky variation. Before image combination, each image was registered and corrected for distortion and rotation with the IRAF tasks "geomap" and "geotran" using a series of unsaturated stars and a polynomial surface fit of order 3. Finally, for each band, the corrected frames were combined with an averaged sigma-clipping algorithm.

The combined science images have been registered on the ICRF frame of reference using the USNO-B1 catalogue. The uncertainty of $0^{\prime \prime} .2$ in the USNO-B1 catalogue dominates the astrometric error, except at the edge of the field of view, where residuals from distortion correction increase the error to about $0^{\prime \prime} .5$. The photometric calibration was performed using several spectrophotometric standard stars from the catalogue of Oke (1990). For the calibration of the NB image we have chosen three standard stars (LDS749B, LTT9491, NGC7293) that did not present any spectral feature in the relevant wavelength range. The derived zero points were consistent with each other within few percent. Magnitudes quoted here are AB magnitudes corrected for galactic extinction (0.09 magnitudes for NB and B band and 0.07 magnitudes for the V band; Schlegel et al. 1998). Given the large number of exposures of the same field at different air-masses we were able to determine the atmospheric extinction coefficients for each night using relatively bright, unsaturated stars.
Table 1: HE0109-3518 field observation log

\begin{tabular}{|c|c|c|c|}
\hline Date & Filter & Seeing $^{a}$ & $t_{\exp }(s)^{b}$ \\
\hline \multirow[t]{3}{*}{2010 Sep. 9} & $\mathrm{~V}_{\text {HIGH }}$ & $0 " .6$ & 5400 \\
\hline & FILT_414_4 & $0 " .7$ & 19500 \\
\hline & $\mathrm{B}_{\mathrm{HIGH}}$ & $0 " .6$ & 720 \\
\hline \multirow[t]{3}{*}{2010 Sep. 10} & $\mathrm{~V}_{\mathrm{HIGH}}$ & $0 " .7$ & 5400 \\
\hline & FILT_414_4 & $0 " .7$ & 21600 \\
\hline & $\mathrm{B}_{\mathrm{HIGH}}$ & $0 " .7$ & 840 \\
\hline \multirow[t]{3}{*}{2010 Sep.11 } & $\mathrm{V}_{\text {HIGH }}$ & $0 " .7$ & 3600 \\
\hline & FILT_414_4 & $0 " .7$ & 11780 \\
\hline & $\mathrm{B}_{\mathrm{HIGH}}$ & $0 " .6$ & 480 \\
\hline \multirow[t]{3}{*}{2010 Sep. 12} & $\mathrm{~V}_{\text {HIGH }}$ & $1 " .3^{c} / 0 " .8$ & 7200 \\
\hline & FILT_414_4 & $0 " .8$ & 19200 \\
\hline & $\mathrm{B}_{\mathrm{HIGH}}$ & $0 " .7$ & 1200 \\
\hline \multirow[t]{3}{*}{ TOTAL } & FILT_414_4 & $0 " .7$ & 72080 \\
\hline & $\mathrm{V}_{\mathrm{HIGH}}$ & $0 " \cdot 7^{d}$ & $21600 / 16200^{d}$ \\
\hline & $\mathrm{B}_{\mathrm{HIGH}}$ & $0 " .7$ & 3240 \\
\hline \multicolumn{4}{|c|}{$\begin{array}{l}a \text { averaged value. } \\
b \text { total exposure time per night and filter. } \\
c \text { average value for the first exposures up to } 5400 \mathrm{~s} . \\
{ }^{d} \text { discarding bad seeing }\left(>1^{\prime \prime}\right) \text { exposures. }\end{array}$} \\
\hline
\end{tabular}

\subsection{Ly $\alpha$ candidate selection}

The selection and photometry of the $\operatorname{Ly} \alpha$ candidates have been performed using an iterative method based on the program SExtractor (Bertin \& Arnouts 1996; v2.5) and IDL procedures. The narrow-band (NB) image alone has been used for candidate detection. Aperture photometry was subsequently performed on both NB and broad-band images using SExtractor dual-mode. This strategy was preferred above a combination of narrow and broad-band as detection image (as sometimes done in the literature for Ly $\alpha$ emitter searches, e.g., Fynbo et al. 2003) since this would bias our results towards objects with continuum-detection, while we are especially interested in detecting object without significant associated continuum.

In order to minimize spurious detections we varied the main detection parameters in SExtractor creating a large number of candidate catalogues. In particular, we used a minimum area for detection (DETECT_MINAREA) ranging from 4 to 8 pixels and a detection threshold (DETECT_THRESH) spanning from 1.3 to 2 times the local noise level. These parameter sets were applied to both unsmoothed images and to images smoothed with two different gaussian kernels. Each SExtractor pass produced, on average, about 3000 detections. The same procedure was applied to detecting candidates in the broad-band images. These were associated by SExtractor, whenever possible, to the objects found in the narrow-band using the dual-image mode. Initial photometry was performed with isophotal apertures (FLUX_ISO) and SExtractor FLUX_AUTO for each band.

As a first step in the selection of $\operatorname{Ly} \alpha$ candidates, we derived an initial guess on the candidate observed EWs from the magnitudes obtained by SExtractor:

$$
E W=\Delta \lambda_{\mathrm{NB}}\left[\frac{\lambda_{\mathrm{V}}}{\lambda_{\mathrm{NB}}}\right]^{\beta_{\lambda}+2} \times\left(10^{-0.4\left(\mathrm{NB}_{\mathrm{AB}}-\mathrm{V}_{\mathrm{AB}}\right)}-1\right) .
$$

Where $\Delta \lambda_{\mathrm{NB}}$ and $\lambda_{\mathrm{NB}}$ are, respectively, the NB filter FWHM and central wavelength, $\lambda_{\mathrm{V}}$ is the $\mathrm{V}_{\mathrm{HIGH}}$ filter effective central wavelength, $\beta_{\lambda}$ is the slope of the UV continuum (in units of $\mathrm{erg} \mathrm{s}^{-1} \mathrm{~cm}^{-2} \AA$ ) between $\lambda_{\mathrm{V}}$ and $\lambda_{\mathrm{NB}}, \mathrm{NB}_{\mathrm{AB}}$ and 
$\mathrm{V}_{\mathrm{AB}}$ are, respectively, the $\mathrm{NB}$ and $\mathrm{V}$ magnitude obtained by Sextractor. We used the V-band to estimate the continuum since it is not affected by the presence of the Ly $\alpha$ emission line. With this strategy we are able to better select objects without significant continuum emission. At the same time, this simplifies the calculation of the EW. When both are present, we used the V-band and B-band isophotal magnitude to derive $\beta_{\lambda}$, otherwise we assigned to the source a flat continuum slope (in frequency), i.e. $\beta_{\lambda}=-2$. This is the typical maximal value for a starburst galaxy (e.g., Meurer et al. 1999). Ly $\alpha$ surveys at $2.1<z<2.3$ find $\beta_{\lambda} \sim-1.9$ for the most luminous sources and higher values $\left(\beta_{\lambda} \sim-1.5\right.$ but with a large scatter) for the faintest (e.g., Guaita et al. 2011, Nilsson et al. 2009). Detected objects with $\beta_{\lambda}<-3$ are extremely rare. Note that a value of $\beta_{\lambda}=-3$ would only imply a decrease of about $25 \%$ of our estimated EW. For the objects without broad-band detection we assigned an initial continuum flux corresponding to the average $2 \sigma$ level within a 1" photometric aperture across the image (larger than the typical seeing of $0^{\prime \prime} .7$ ). To convert the NB magnitude to a line flux, we assumed that the emission line is fully contained by the NB filter and centered at the filter transmission peak 2 .

Since the filter transmission curve has a roughly gaussian shape, the derived flux is thus a lower limit to the actual emission. This implies that the quoted EW should also be considered as a lower limit, strengthening the results discussed in section 3 . We did not attempt to remove the line contribution from the B-band magnitude used to estimate $\beta_{\lambda}$. This is also a conservative assumption for the estimation of the EW.

For each catalogue we then performed a first cut selecting objects with observed $\mathrm{EW}>68 \AA$, corresponding to a rest-frame $\mathrm{EW}_{0}=\mathrm{EW} /(1+z)>20 \AA$ for Ly $\alpha$ at $z=2.4$. We chose this $\mathrm{EW}_{0}$ cut in order to be comparable with the majority of $\operatorname{Ly} \alpha$ surveys in the literature. This produced Ly $\alpha$ candidate catalogues of which the largest contained 224 objects. Then we cleaned through visual inspection the spurious detections due to the vicinity of bright stars, excessive fragmenting of objects (e.g., galaxies showing multiple spatial components in the narrow-band and a single spatial component in the broad-band) and noise at the edges of the image. This produced a set of catalogues containing a number of candidates varying from 89 to 100 .

For the objects in the cleaned catalogues without Sextractor broad-band detections we performed again photometry using our own specifically designed IDL procedures in order to derive an upper limit on the broad-band flux and therefore a lower limit on the EW. The sources without Sextractor broad-band detection are typically spatially compact or unresolved in the NB image. Therefore we used circular apertures with a fixed size of 1.5 arcsec (twice the seeing disk, 0.7", that is coincidentally the same for each band) for both the NB and broad-bands. Using exactly the same aperture for NB and broad-band is again a conservative assumption for the calculation of the EW, since the gas extended

2 This is a similar approach with respect to the one used by other deep surveys not targeting a bright quasar (e.g., Grove et al. 2009, Hayes et al. 2010) that we will use in section 4 for a direct comparison of the $\operatorname{Ly} \alpha$ luminosity functions.

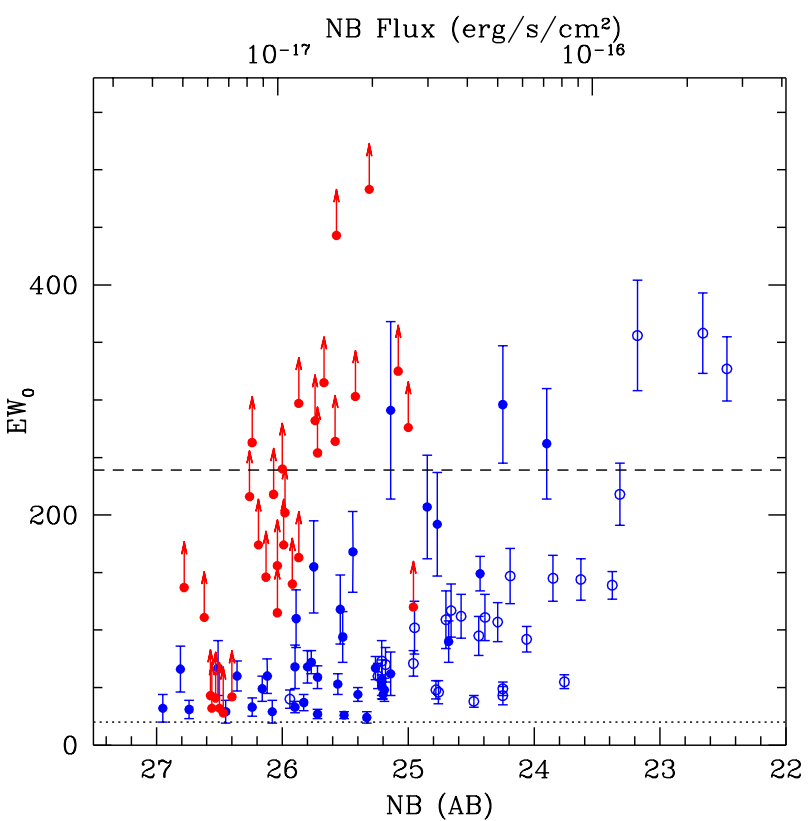

Figure 1. Rest frame equivalent width $\left(\mathrm{EW}_{0}\right)$ of the detected objects versus their narrow-band magnitudes. Blue solid (or open) circles represent candidates with V-band (or both V and B-band) detections above the $3 \sigma$ level for $\mathrm{V}$-band (and $5 \sigma$ for the B-band). When the B-band is detected (open circles) the $\mathrm{EW}_{0}$ is derived from a continuum conservatively estimated from both $\mathrm{V}$ and Bbands without correcting for the line contribution to the B-band. For objects without $\mathrm{B}$ detection, we estimate the continuum from the V-band, assuming a continuum slope of $\beta_{\lambda}=-2$. The red solid circles represent lower limits on the $\mathrm{EW}_{0}$ of the objects without continuum detection (i.e., $\mathrm{V}<3 \sigma$ ). These correspond to the (local) $1 \sigma$ lower limits for objects with signal to noise ratio $\mathrm{SNR}<0$ in V-band (the deepest available), otherwise the measured flux plus the local $1 \sigma$ noise has been used (e.g., Feldman $\&$ Cousins 1998; see text for details). The dashed line represents the theoretical limit on the $\mathrm{EW}_{0}$ that is produced by "normal" stellar populations $\left(\mathrm{EW}_{0}=240 \AA\right.$; e.g., Charlot \& Fall 1993 , Schaerer 2002) while the dotted line is the lowest $\mathrm{EW}_{0}$ for candidate selection $\left(\mathrm{EW}_{0}=20 \AA\right.$, consistent with most published Ly $\alpha$ surveys). The continuum-undetected objects with $\mathrm{EW}_{0}>240 \AA$ (red dots above the dashed line) are the best candidates for protogalactic, or "dark", gas-rich clouds that have been fluorescently illuminated by the quasar.

distribution and scattering effects would likely result in a Ly $\alpha$ emission that is more extended than the stellar continuum (e.g., Cantalupo et al. 2005; see also Steidel et al. 2011). The apertures were centered, for all bands, at the location corresponding to the peak of the NB emission (derived from SExtractor). We used relative astrometry to refine the position of these apertures in the broad-bands. If the flux measured in the broad-band apertures was above 3 times the local noise level $(\sigma)$ we considered the source continuumdetected and we used the measured flux to compute the EW. Otherwise, we consider the object undetected in the continuum and we estimate an upper limit on the continuum flux using a procedure similar to Feldman \& Cousins (1998). For apertures where the measured flux is positive this corresponds to assigning an upper limit equal to the sum of the measured value and the local $1 \sigma$. In case the flux 
is negative, although Feldman \& Cousins (1998) provide a table for assigning upper limits (with values $<1 \sigma$ ) in this situation, we decided to use as a conservative value the local $1 \sigma$ as a minimum threshold. The continuum flux upper limits translates then into EW lower limits. With this method, we use the statistical information contained in the measured flux and we have a consistent measurement with the $1 \sigma$ errorbars of the detected objects, i.e. we make sure that the actual $\mathrm{EW}$ is above the reported value within at least $1 \sigma$ confidence level. We note that current Ly $\alpha$ surveys in literature use different methods to estimate continuum flux upper limits for undetected sources. In particular, a common choice is to use a fix value (e.g., a globally estimated $1 \sigma$ or $2 \sigma$ value for a given aperture) independently of the measured flux. This complicates the comparison of different works in the literature.

Bright foreground objects in proximity to the aperture were masked, but we cannot rule out the possibility that fainter foreground objects are contaminating the apertures in the broad-band. Note that this would imply that the derived EW of the objects in question are underestimated and thereby strengthen the conclusions presented in the paper. For the objects with SExtractor broad-band detection we used FLUX_AUTO to calculate total magnitudes and isophotal apertures (FLUX_ISO) to compute the colors and EW, as commonly performed in previous Ly $\alpha$ searches (e.g., Hayes et al. 2010). Isophotal apertures and FLUX_AUTO measurement did not produce magnitudes varying more than few percent from each other, except in the few cases where sources were located close to foreground objects. Only objects with a $\mathrm{SNR} \gtrsim 5$ in the narrowband images were retained in the catalogues. This corresponds to about $\mathrm{NB}_{\mathrm{AB}}(5 \sigma) \sim 26.8$ on average across the image, while the typical (median) $1 \sigma$ magnitude limits for $\mathrm{V}$-band and B-band were, respectively, $\mathrm{V}_{\mathrm{AB}}(1 \sigma) \sim 29.7$ and $\mathrm{B}_{\mathrm{AB}}(1 \sigma) \sim 29.1$ for a 1.5 " diameter circular aperture.

In order to assess the level of spurious detections due to noise for each set of parameters and filters we produced a negative catalogue applying SExtractor to the narrow-band image multiplied by -1 . The number of detected objects in the negative catalogues varied from zero to 30 . In the latter case, however, these spurious detections were mostly confined at the image edges, while only between three to five spurious objects were present at the center. Among the eleven candidates that were not present in every positive catalogue, nine did not have any broad-band detection. For these nine candidates we have rechecked the narrow-band image splitting the total exposure time in four parts (each corresponding to a given rotation angle). Six objects were detectable (at SNR $>3$ ) in at least three out of four images. The remaining three objects, in accordance with the number found in the negative catalogues, were considered spurious and thus removed from their catalogues. The final catalogue contained thus 97 sources. In section 6.1 we discuss in detail the possible fraction of foreground contaminations in our sample.

Finally, we estimated the completeness of our NB imaging with the standard procedure of distributing and recovering a large number $(\sim 1000)$ of artificial sources with varying sizes. In particular, we used the distribution of apertures derived by SExactror for our detected candidates obtaining a completeness factor $f_{c}=(25 \%, 50 \%, 75 \%, 90 \%)$ for
$N B=(26.95,26.55,26.30,26.12)$ corresponding to Ly $\alpha$ luminosities, at $z=2.4, L_{\mathrm{Ly} \alpha}=(0.20,0.30,0.38,0.43)$ in units of $10^{42} \mathrm{erg} \mathrm{s}^{-1}$. The factor $f_{c}$ will be used to correct the Ly $\alpha$ luminosity function for incompleteness in section 4.2

\section{RESULTS}

The final, merged catalogue consists of 97 narrow-band excess objects. The position and full properties of all detected objects are reported in tabular form in Tables 3] 3] and Appendix A. The EW (estimated as in section 2.3 ) and NB magnitudes of these objects are presented in Figure 1. Among the 97 NB-detected objects, 31 do not have any detectable continuum (above $3 \sigma$ ) in the very deep broad-band images (red symbols with arrows in Figure 1) and therefore the reported EW has to be considered a lower limit as discussed in section 2.3 Given the sensitivity of our broadband imaging we are able to put strong constraints on the EW limits, at least for the objects with NB magnitudes $\mathrm{NB}_{A B}<26$. Among these, $12 \mathrm{Ly} \alpha$ candidates have a lower limit $\mathrm{EW}_{0}>240 \AA$, i.e. higher than the typical maximum value produced by normal stellar populations (e.g., Charlot \& Fall 1993, Schaerer 2002; see also Malhotra \& Rhoads 2002 and Raiter, Schaerer \& Fosbury 2010). Six of the 66 objects that have at least one broad-band detection (blue circles in Figure 1) also have $\mathrm{EW}_{0}>240 \AA$. As we will show in this section, these contain also the objects with the most evident signs of extended, filamentary emission.

In Figure 2 we present the combined narrow-band image (20 hours combined integration time) with overlaid positions of the 97 Ly $\alpha$ candidates. The quasar is near the center of the image. Open squares (circles) represent objects with a measured or lower limit $\mathrm{EW}_{0}>240 \AA\left(\mathrm{EW}_{0}<240 \AA\right)$ while red (or blue) colors indicate if the object was undetected (or detected) in any of the broad-band images.

From the image, we have visually identified an additional narrow-band excess source, i.e., a blob-like extended emission (magenta ellipse in Figure 2 approximatively at $R A=1: 11: 57$ and $\delta=-35: 01: 50)$. Due to the presence of relatively bright components within this extended image, which appear also in the broad-band (possibly, foreground objects), this extended source was split into several parts by SExtractor and it did not make it to the final catalogue using our selection technique. We will refer to this object as LAB1 from now on.

In Figure 3. we show postage stamp images of 12 candidates without continuum detection $(V<3 \sigma)$ and lower limit $\mathrm{EW}_{0}>240 \AA$. The objects are ordered by decreasing EW limit from the top to the bottom panels. Their positions, photometric properties and EW limits are reported in Table 3 Among the brightest candidates, \#1484 and \#902 have a very high lower limit on their rest-frame Ly $\alpha$ equivalent widths, namely $\mathrm{EW}_{0}>>400 \AA$. Interestingly, \#1484 is also the source closest to the quasar (in projection), located just about 15 arcsec away (the quasar is just above the top right corner in the first panel of Figure 3). Note that the vast majority of the objects without broad-band detection appear spatially compact.

In Figure 4, we show a stacked NB (left-hand panel) and V-band (right-hand panel) image of the 12 protogalactic candidates, i.e. the continuum-undetected sources 


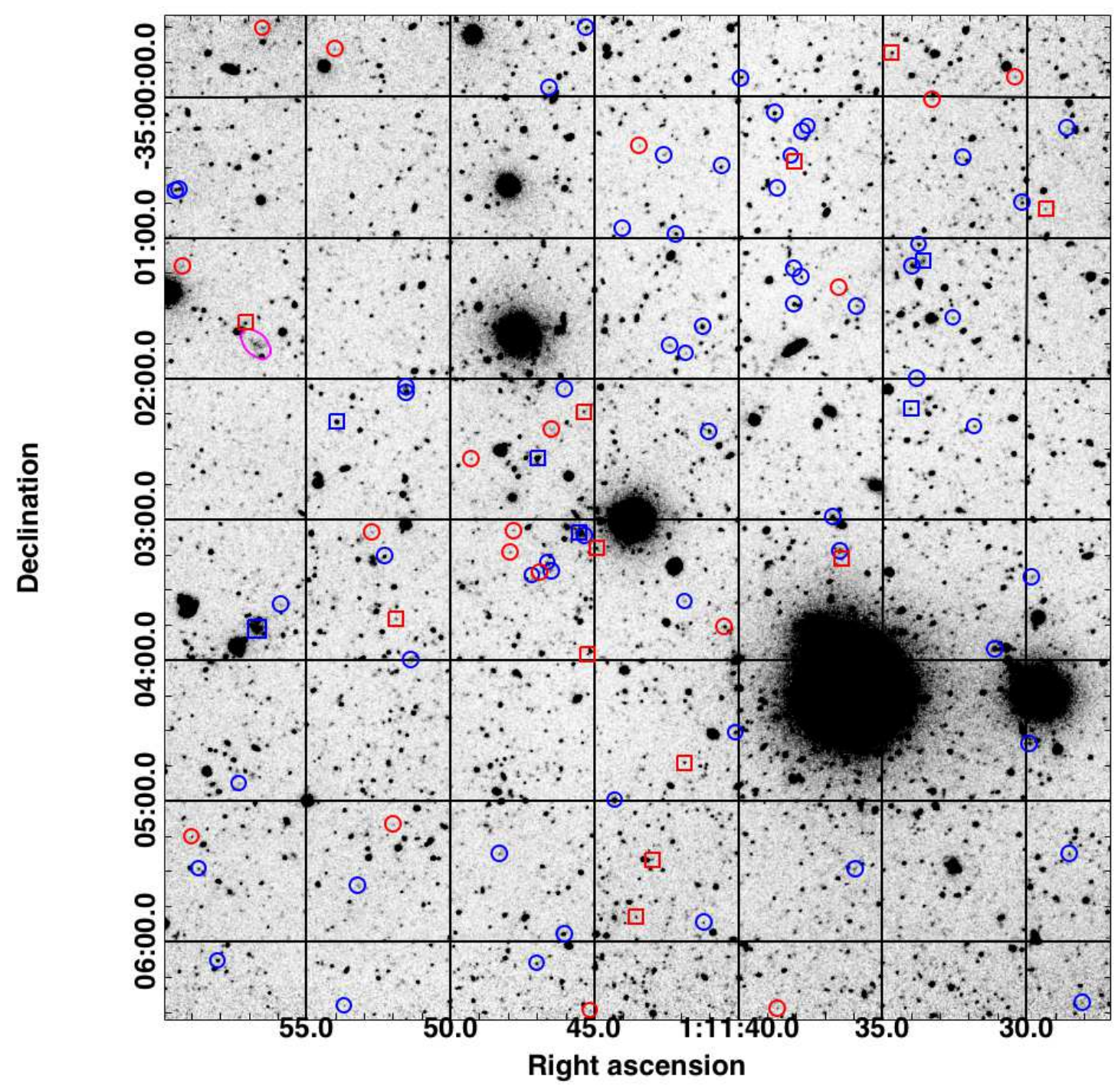

Figure 2. The combined narrow-band image (20 hours total integration time) of the field surrounding HE0109-3518 (near the center of the image). Squares (or circles) indicate the position of detected emission line objects with $\mathrm{EW}_{0}>240 \AA$ (or $\mathrm{EW}_{0}<240 \AA$ ), assuming the line is Ly $\alpha$. Red (or blue) colors represent objects undetected (or detected) in the broad-band images. The position of the extended, blob-like emission source (LAB1) is indicated by a magenta ellipse (approximatively at $r a=1: 11: 57$ and dec = $-35: 01: 50$ ). Objects with undetected continuum and with $\mathrm{EW}_{0}>240 \AA$ (i.e. the red squares) are the best candidates for protogalactic clouds, or "dark" galaxies, fluorescently illuminated by the quasar (see text for details).

with $\mathrm{EW}_{0}>240 \AA$. Before stacking, bright sources have been masked everywhere except in the central 3"-wide region (indicated by the black circle) and the local (30" radius), sigmaclipped mean has been subtracted from each image. We then performed aperture photometry within a 2" diameter aperture on both the NB and V-band stack, centered on the position derived from the NB. The measured flux in V-band is below the $1 \sigma$ level for a 2 " aperture, i.e. $V_{\text {stack }} \sim 30.3$, implying a combined constraint of $\mathrm{EW}_{0, \text { stack }}>800 \AA(1 \sigma)$. This measurement strongly suggests that internal stellar sources, if present at all, do not contribute significantly to the Ly $\alpha$ emission 3 . The stacked Ly $\alpha$ emission profile remains com-

\footnotetext{
3 As a reference, we have estimated with CLOUDY (v10.00; Ferland et al. 1998) that an $\mathrm{EW}_{0}>800 \AA$ is still one order of magnitude lower than the value expected from pure nebular continuum if measured at rest-frame $1600 \AA$ and using $\beta_{\lambda}=-2$ for a fully ionized cloud by a typical quasar spectrum $(\alpha=-1.7$; e.g. Telfer et al. 2002).
} 




Figure 3. Images of the narrow-band excess sources without continuum detection $(V<3 \sigma)$ and $\mathrm{EW}_{0}>240 \AA$ (assuming the line is $\mathrm{Ly} \alpha$ ) in three different bands: narrow-band (left-hand panels), B (central panels), and V (right-hand panels). For an object at $z=2.4$, the central wavelength of these filters correspond to the rest-frame Ly $\alpha, 1300 \AA$ and $1600 \AA$, respectively. Note that the B-band contains also the source Ly $\alpha$ line, differently from the V-band, although the emission would be typically too faint to be detected in B. Panel dimensions are $15 \times 15 \mathrm{arc} \mathrm{sec}^{2}$. The images have been smoothed with a gaussian kernel with radius 0 ".5 (two pixels) for clarity. These objects represent the best candidates for fluorescent protogalactic clouds or "dark" galaxies given the absence of detected continuum and the high lower limit on the $\mathrm{EW}_{0}$. As discussed in the text, an $\mathrm{EW}_{0}>240 \AA$ strongly suggests that internal star formation is not likely the origin of the Ly $\alpha$ emission and that we are detecting dense gas fluorescently illuminated by the quasar. 

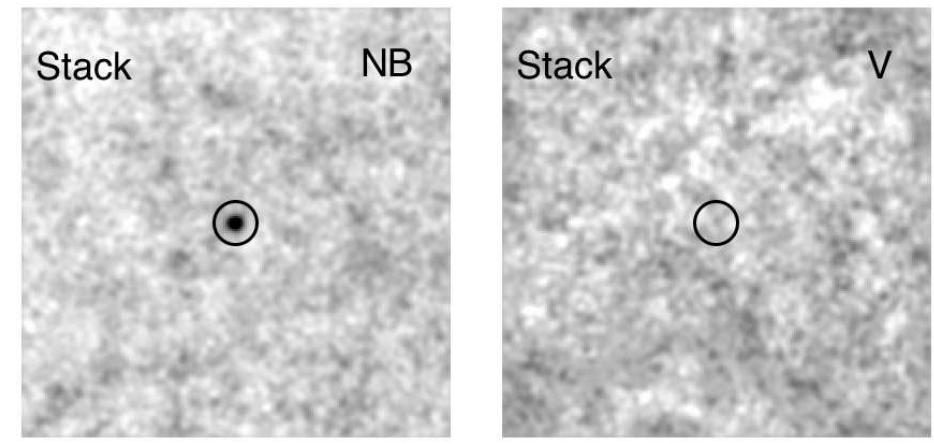

Figure 4. Stacked NB image (left-hand panel) and V-band image (right-hand panel) of the 12 Ly $\alpha$ sources that did not have individual continuum detection and an implied $\mathrm{EW}_{0}>240 \AA$. The images have been smoothed with a gaussian kernel with radius 0 ". 5 (two pixels) for clarity. The V-band stack does not show any continuum source above $1 \sigma$ level (V 30.3). The combined constraint implies a $\mathrm{EW}_{0}>800 \AA$, effectively ruling out internal star-formation as the origin of the Ly $\alpha$ emission in these objects.

pact and consistent with a point source with FWHM size of about 1" (about 8 physical kpc at $z=2.4$ ).

Postage stamp images and summary properties of the Ly $\alpha$ candidates with broad-band detections $(>3 \sigma)$ and measured $\mathrm{EW}_{0}>240 \AA$ are presented in Figure 5 and Table 3. respectively. It can be seen that the three brightest Ly $\alpha$ candidates in our sample, \#1159, \#2886 and \#1412 are also the objects with the highest measured EW, i.e. $\mathrm{EW}_{0} \sim 350 \AA$. They are detected both in $\mathrm{V}$ and $\mathrm{B}$ bands and show a very red $V-B$ color. In particular, their $\mathrm{B}$ magnitudes are faint enough that the very bright Ly $\alpha$ emission could contribute most of the broad-band flux. The presence of a stellar continuum is favored by the clearly detected flux in V-band, but either the stellar population is old or it is embedded in a large amount of dust that is not affecting the Ly $\alpha$ emission. This would be necessary to reconcile the red color with the high $\mathrm{Ly} \alpha \mathrm{EW}$. As we will discuss in section 5.3, a possible explanation is that quasar fluorescence is illuminating a large, dust-poor gas reservoir - the Circum Galactic Medium (CGM) - surrounding dust-rich, star-forming galaxies.

At least three objects, \#1159, \#1412 and \#2411, show evidence of extended, possibly filamentary, emission. In particular, the image of \#1159 shows a hint of three structures extending out of the bright nucleus. The longest of these has a (projected) linear size of about 4", corresponding to about 30 physical $\mathrm{kpc}$ at $z=2.4$. This scale is similar to the virial radius of $\mathrm{a} \sim 10^{11} M_{\odot}$ dark-matter halo at this redshift. Object \#2411 also shows a filamentary "bridge" on similar (projected) scales apparently connecting one region of extended emission without a compact nucleus (and with only a marginal detection in V-band) to another bright narrowband excess object with high EW (\#2433). Revealing the nature of these two apparently connected Ly $\alpha$ companions is complicated by the fact that the broad-band images show a continuum source between them. Without knowing the redshift and the spectral energy distribution of this continuum object, it is difficult to estimate its contribution to the NB observed flux. However, the red $B-V$ color and the different morphology argue that such compact object cannot be the only source of narrow-band emission in the "bridge". If \#2411 and \#2433 are physically connected, this emitting "bridge" may have a similar origin to the narrow gas filament discovered by Rauch et al. (2011) in a deep blind spectroscopic survey at $z=3.3$ and corresponding to a continuum source showing tidal tails in HST imaging.

In Figure 6] we show the narrow and broad-band postage stamp images of the blob-like extended emitter LAB1. Morphologically, this object resembles other elongated Ly $\alpha$ blobs previously discovered (e.g., Steidel et al. 2000, Matsuda et al. 2004, Nilsson et al. 2006, Yang et al. 2009, Prescott et al. 2012). It shows a relatively uniform surface brightness except for one peak, which is off centered with respect to the extended emission. At least four continuum sources are detected in the broad-band image(s) within the projected area of LAB1. One of these sources is located in the proximity of the NB emission peak. The overall shape of LAB1 seems to follow these continuum objects but at present we are not able to confirm if they lie at the same redshift as LAB1. Note that the bright NB excess object in the top-left corner in Figure 6 (about 10" away from LAB1) is \#2620, one of the objects without continuum-detection with high $\mathrm{EW}$ limit $\left(\mathrm{EW}_{0}>276 \AA\right)$.

\section{PHYSICAL ORIGIN OF THE EMISSION}

In this section, we compare our observational results to those obtained in similar deep searches for $\operatorname{Ly} \alpha$ emission in regions that do not target high-redshift quasars (we refer to these surveys as "blank-field" studies) and with the results of radiative-transfer simulations. In particular, we will examine the EW distributions, Luminosity Functions and the relation between (projected) distances form the quasar and candidate luminosities. The combination of these statistics provide strong evidence for the fluorescent origin of the line emission of many of the objects in our sample.

\subsection{EW distribution}

The Ly $\alpha$ EW is one of the most important diagnostics to distinguish between internal star-formation and other mechanisms that can produce Ly $\alpha$ emission (e.g., Schaerer 2002), 
Table 2: Position and properties of Ly $\alpha$ candidates without continuum detection $(V$ and $B<3 \sigma)$

\begin{tabular}{|c|c|c|c|c|c|c|c|c|c|}
\hline Id & $\begin{array}{c}\text { RA } \\
(\mathrm{J} 2000)\end{array}$ & $\begin{array}{c}\text { DEC } \\
(\mathrm{J} 2000)\end{array}$ & $\begin{array}{l}\mathrm{NB}^{a} \\
(\mathrm{AB})\end{array}$ & $\begin{array}{l}\mathrm{V}_{l i m}^{b} \\
(\mathrm{AB})\end{array}$ & $\operatorname{SNR}(\mathrm{V})^{c}$ & $\begin{array}{l}\mathrm{B}_{l i m}^{b} \\
(\mathrm{AB})\end{array}$ & $\underset{10^{-17} \mathrm{erg} \mathrm{s}^{-1} \mathrm{~cm}^{-2}}{d}$ & $\begin{array}{c}\mathrm{L}_{\text {Ly }}^{e} \\
10^{42} \mathrm{erg} \mathrm{s}^{-1}\end{array}$ & $\begin{array}{c}\mathrm{EW}_{0}^{f} \\
\AA\end{array}$ \\
\hline 1484 & $1: 11: 44.83$ & $-35: 03: 12.1$ & $25.31_{-0.08}^{+0.09}$ & $>29.71$ & -1.1 & $>28.16$ & $1.95 \pm 0.16$ & $0.91 \pm 0.07$ & $>483$ \\
\hline 902 & $1: 11: 41.80$ & $-35: 04: 43.5$ & $25.57_{-0.14}^{+0.15}$ & $>29.75$ & -0.8 & $>29.45$ & $1.54 \pm 0.20$ & $0.71 \pm 0.09$ & $>443$ \\
\hline 1737 & $1: 11: 34.45$ & $-34: 59: 41.9$ & $25.08_{-0.08}^{+0.08}$ & $>29.22$ & 0.5 & $>29.38$ & $2.42 \pm 0.17$ & $1.12 \pm 0.08$ & $>325$ \\
\hline 390 & $1: 11: 43.50$ & $-35: 05: 48.7$ & $25.67_{-0.13}^{+0.14}$ & $>29.75$ & -1.5 & $>29.41$ & $1.40 \pm 0.17$ & $0.65 \pm 0.08$ & $>315$ \\
\hline 2944 & $1: 11: 45.28$ & $-35: 02: 14.1$ & $25.42_{-0.10}^{+0.11}$ & $>29.69$ & -0.3 & $>29.38$ & $1.77 \pm 0.17$ & $0.82 \pm 0.08$ & $>303$ \\
\hline 1321 & $1: 11: 51.76$ & $-35: 03: 42.0$ & $25.87_{-0.15}^{+0.10}$ & $>29.78$ & -0.3 & $>29.15$ & $1.17 \pm 0.17$ & $0.54 \pm 0.08$ & $>297$ \\
\hline 555 & $1: 11: 42.91$ & $-35: 05: 24.5$ & $25.74_{-0.11}^{+0.12}$ & $>29.80$ & -0.6 & $>29.47$ & $1.32 \pm 0.14$ & $0.61 \pm 0.06$ & $>282$ \\
\hline 2620 & $1: 11: 57.01$ & $-35: 01: 35.9$ & $25.00_{-0.07}^{+0.07}$ & $>29.12$ & 0.8 & $>28.62$ & $2.60 \pm 0.16$ & $1.21 \pm 0.08$ & $>276$ \\
\hline 2131 & $1: 11: 37.91$ & $-35: 00: 27.9$ & $25.58_{-0.10}^{+0.11}$ & $>29.51$ & 0.3 & $>29.54$ & $1.52 \pm 0.15$ & $0.71 \pm 0.07$ & $>264$ \\
\hline 2274 & $1: 11: 29.17$ & $-35: 00: 47.3$ & $26.24_{-0.14}^{+0.16}$ & $>29.86$ & -1.8 & $>29.54$ & $0.83 \pm 0.11$ & $0.39 \pm 0.05$ & $>263$ \\
\hline 1519 & $1: 11: 36.36$ & $-35: 03: 17.0$ & $25.72_{-0.14}^{+0.15}$ & $>29.62$ & -0.9 & $>29.31$ & $1.34 \pm 0.17$ & $0.62 \pm 0.08$ & $>254$ \\
\hline 1212 & $1: 11: 45.14$ & $-35: 03: 57.1$ & $26.00_{-0.09}^{+0.10}$ & $>29.49$ & 0.3 & $>27.91$ & $1.04 \pm 0.09$ & $0.48 \pm 0.04$ & $>240$ \\
\hline 1498 & $1: 11: 47.81$ & $-35: 03: 13.8$ & $26.07_{-0.16}^{+0.17}$ & $>29.71$ & -1.6 & $>29.43$ & $0.97 \pm 0.14$ & $0.45 \pm 0.07$ & $>218$ \\
\hline 2842 & $1: 11: 49.17$ & $-35: 02: 34.0$ & $26.26_{-0.15}^{+0.10}$ & $>29.68$ & -1.5 & $>29.39$ & $0.81 \pm 0.12$ & $0.38 \pm 0.05$ & $>216$ \\
\hline 1829 & $1: 11: 30.15$ & $-34: 59: 51.5$ & $25.98_{-0.14}^{+0.15}$ & $>29.80$ & -0.1 & $>28.50$ & $1.05 \pm 0.14$ & $0.49 \pm 0.06$ & $>202$ \\
\hline 2985 & 1:11:46.39 & $-35: 02: 21.5$ & $25.99_{-0.13}^{+0.14}$ & $>29.69$ & -0.1 & $>29.38$ & $1.04 \pm 0.13$ & $0.49 \pm 0.06$ & $>174$ \\
\hline 1565 & $1: 11: 46.79$ & $-35: 03: 22.1$ & $26.19_{-0.16}^{+0.17}$ & $>29.71$ & -3.0 & $>29.44$ & $0.87 \pm 0.13$ & $0.40 \pm 0.06$ & $>174$ \\
\hline 1926 & 1:11:33.06 & $-35: 00: 01.2$ & $25.87_{-0.12}^{+0.13}$ & $>29.47$ & 0.4 & $>28.38$ & $1.17 \pm 0.13$ & $0.54 \pm 0.06$ & $>163$ \\
\hline 619 & $1: 11: 58.87$ & $-35: 05: 16.2$ & $26.04_{-0.14}^{+0.15}$ & $>29.26$ & 0.6 & $>29.54$ & $1.00 \pm 0.13$ & $0.46 \pm 0.06$ & $>156$ \\
\hline 2034 & $1: 11: 43.31$ & $-35: 00: 21.3$ & $26.13_{-0.14}^{+0.15}$ & $>29.57$ & 0.2 & $>29.52$ & $0.92 \pm 0.12$ & $0.43 \pm 0.06$ & $>146$ \\
\hline 1706 & $1: 11: 56.37$ & $-34: 59: 30.7$ & $25.92_{-0.14}^{+0.16}$ & $>29.39$ & -0.2 & $>28.50$ & $1.11 \pm 0.15$ & $0.52 \pm 0.07$ & $>140$ \\
\hline 85 & $1: 11: 38.64$ & $-35: 06: 27.6$ & $26.78_{-0.19}^{+0.22}$ & $>29.59$ & -1.0 & $>29.04$ & $0.50 \pm 0.09$ & $0.23 \pm 0.04$ & $>137$ \\
\hline 1279 & $1: 11: 40.40$ & $-35: 03: 45.7$ & $24.96_{-0.09}^{+0.09}$ & $>28.26$ & 2.7 & $>29.39$ & $2.70 \pm 0.21$ & $1.25 \pm 0.10$ & $>120$ \\
\hline 1439 & $1: 11: 52.60$ & $-35: 03: 05.2$ & $26.04_{-0.13}^{+0.13}$ & $>29.78$ & -0.3 & $>29.06$ & $1.00 \pm 0.11$ & $0.46 \pm 0.05$ & $>115$ \\
\hline 2454 & $1: 11: 59.18$ & $-35: 01: 11.8$ & $26.62_{-0.19}^{+0.19}$ & $>29.52$ & -0.2 & $>28.62$ & $0.58 \pm 0.11$ & $0.27 \pm 0.05$ & $>111$ \\
\hline 70 & $1: 11: 45.10$ & $-35: 06: 28.1$ & $26.57_{-0.21}^{+0.19}$ & $>28.58$ & 1.0 & $>28.93$ & $0.61 \pm 0.13$ & $0.28 \pm 0.06$ & $>43$ \\
\hline 1432 & 1:11:47.69 & $-35: 03: 04.5$ & $26.40_{-0.20}^{+0.23}$ & $>28.21$ & 2.9 & $>29.43$ & $0.72 \pm 0.14$ & $0.33 \pm 0.06$ & $>42$ \\
\hline 1762 & $1: 11: 53.86$ & $-34: 59: 39.6$ & $26.53_{-0.19}^{+0.21}$ & $>28.53$ & 1.6 & $>29.27$ & $0.64 \pm 0.11$ & $0.30 \pm 0.05$ & $>41$ \\
\hline 2525 & $1: 11: 36.41$ & $-35: 01: 21.3$ & $26.50_{-0.18}^{+0.19}$ & $>28.41$ & 2.7 & $>29.50$ & $0.65 \pm 0.11$ & $0.30 \pm 0.05$ & $>32$ \\
\hline 665 & $1: 11: 51.87$ & $-35: 05: 09.6$ & $26.56_{-0.17}^{+0.19}$ & $>28.37$ & 2.9 & $>29.53$ & $0.62 \pm 0.10$ & $0.29 \pm 0.05$ & $>32$ \\
\hline 1989 & $1: 11: 38.01$ & $-35: 00: 25.4$ & $26.47_{-0.25}^{+0.31}$ & $>27.82$ & 2.8 & $>28.40$ & $0.67 \pm 0.17$ & $0.31 \pm 0.08$ & $>28$ \\
\hline
\end{tabular}

${ }^{a}$ Total magnitude.

${ }^{b}$ Lower limits - measured value plus the local $1 \sigma$ or local $1 \sigma$ if SNR $<0$ - derived from a circular aperture of 1 ".5 diameter.

${ }^{c}$ V-band Signal to Noise Ratio within a circular aperture of 1".5 diameter.

${ }^{d}$ Derived from total NB magnitudes assuming that emission line is at the filter central wavelength (lower limits).

$e^{e}$ Derived from $\mathrm{F}_{L y \alpha}$ assuming $\mathrm{z}=2.4$.

${ }^{f}$ Rest frame EW limits within a 1".5 diameter circular aperture estimated from $V_{\text {lim }}$ assuming an UV continuum slope $\beta_{\lambda}=-2$.

Table 3: Position and properties of Ly $\alpha$ candidates with continuum detection $(V>3 \sigma)$ and $\mathrm{EW}_{0}>240 \AA^{a}$

\begin{tabular}{ccccccccc}
\hline \hline Id & $\begin{array}{c}\text { RA } \\
(\mathrm{J} 2000)\end{array}$ & $\begin{array}{c}\mathrm{DEC} \\
(\mathrm{J} 2000)\end{array}$ & $\begin{array}{c}\mathrm{NB} \\
(\mathrm{AB})\end{array}$ & $\begin{array}{c}\mathrm{V}^{b} \\
(\mathrm{AB})\end{array}$ & $\begin{array}{c}\mathrm{B}^{b} \\
(\mathrm{AB})\end{array}$ & $\begin{array}{c}\mathrm{F}_{L y \alpha}^{c} \\
10^{-17} \mathrm{erg} \mathrm{s}^{-1} \mathrm{~cm}^{-2}\end{array}$ & $\begin{array}{c}\mathrm{L}_{L y \alpha}^{d} \\
10^{42} \mathrm{erg} \mathrm{s}^{-1}\end{array}$ & $\begin{array}{c}\mathrm{EW}_{0}^{e} \\
\AA\end{array}$ \\
\hline 1159 & $1: 11: 56.59$ & $-35: 03: 46.9$ & $22.66_{-0.04}^{0.04}$ & $24.74_{-0.04}^{+0.04}$ & $26.06_{-0.12}^{+0.13}$ & $22.44 \pm 0.81$ & $10.42 \pm 0.38$ & $358 \pm 35$ \\
2886 & $1: 11: 46.88$ & $-35: 02: 33.8$ & $23.18_{-0.04}^{0.04}$ & $25.99_{-0.06}^{+0.07}$ & $26.72_{-0.15}^{+0.17}$ & $13.90 \pm 0.50$ & $6.46 \pm 0.23$ & $356 \pm 48$ \\
1412 & $1: 11: 45.42$ & $-35: 03: 05.3$ & $22.47_{-0.04}^{+0.04}$ & $25.45_{-0.06}^{+0.06}$ & $25.98_{-0.11}^{+0.11}$ & $26.73 \pm 0.97$ & $12.42 \pm 0.45$ & $327 \pm 28$ \\
2411 & $1: 11: 33.48$ & $-35: 01: 09.8$ & $24.25_{-0.06}^{+0.06}$ & $27.81_{-0.18}^{+0.22}$ & $>28.73$ & $5.19 \pm 0.28$ & $2.41 \pm 0.13$ & $296 \pm 51$ \\
2914 & $1: 11: 33.93$ & $-35: 02: 13.0$ & $25.14_{-0.08}^{+0.08}$ & $28.69_{-0.27}^{+0.34}$ & $>28.52$ & $2.29 \pm 0.16$ & $1.06 \pm 0.08$ & $291 \pm 77$ \\
2936 & $1: 11: 53.84$ & $-35: 02: 18.1$ & $23.90_{-0.05}^{+0.05}$ & $27.34_{-0.2}^{+0.24}$ & $>27.98$ & $7.16 \pm 0.32$ & $3.33 \pm 0.15$ & $262 \pm 48$ \\
\hline $2433^{f}$ & $1: 11: 33.85$ & $-35: 01: 12.4$ & $23.32_{-0.08}^{+0.09}$ & $25.35_{-0.11}^{+0.12}$ & $26.3_{-0.1}^{+0.11}$ & $12.22 \pm 0.97$ & $5.68 \pm 0.45$ & $218 \pm 27$ \\
\hline
\end{tabular}

${ }^{a}$ See Appendix A for the list of the remaining 61 Ly $\alpha$ candidates with continuum detection.

$b$ Total magnitudes (or lower limits for B-band, see Table 2) .

${ }^{c}$ Assuming that emission line is at the filter central wavelength.

${ }^{d}$ Derived from $\mathrm{F}_{L y \alpha}$ assuming $\mathrm{z}=2.4$.

${ }^{e}$ Rest frame EW for $\mathrm{Ly} \alpha$ at $\mathrm{z}=2.4$. A UV continuum slope $\beta_{\lambda}=-2$ is used for objects undetected in B.

$f$ (Projected) "Companion" of \#2411. 

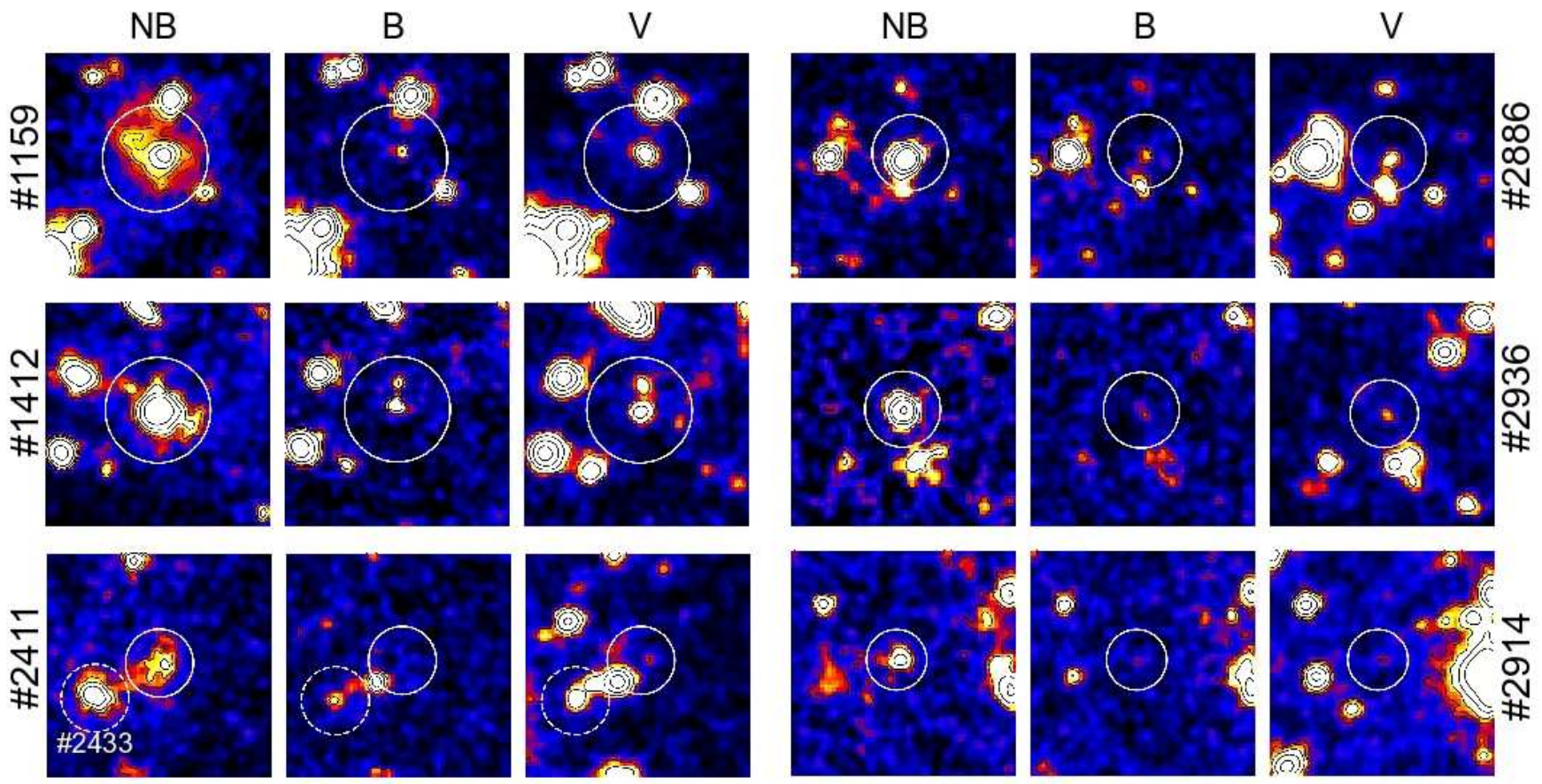

Figure 5. Images of the objects with continuum detection $(V>3 \sigma)$ but still with $\mathrm{EW}_{0}>240 \AA$ (assuming the line is Lyalpha) in three different bands. These sources include the extended objects with filamentary emission (\#1159, \#1412, and \#2411 with its "companion" \#2433). The high value of the $\mathrm{EW}_{0}$ suggests that local star-formation cannot be the only source of the detected radiation. Instead, the emission is fully compatible with dense circum-galactic gas fluorescently illuminated by the quasar (see text for details). Panel dimensions are $15 \times 15 \operatorname{arcsec}^{2}$. The images have been smoothed with a gaussian kernel with radius 0 ". 5 (two pixels) for clarity.

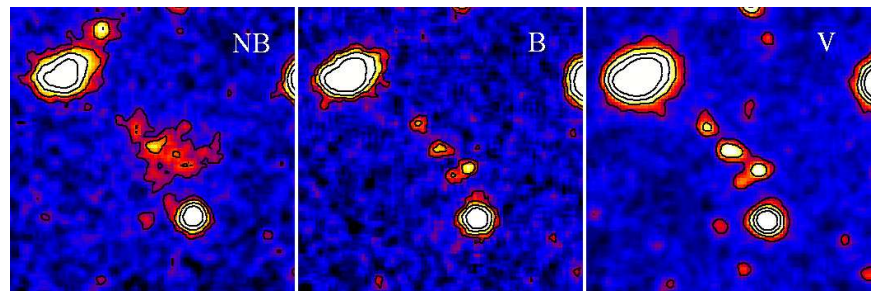

Figure 6. Postage stamp images of the extended, blob-like emission LAB1 in narrow-band (left-hand panel), B-band (central panel) and V-band (right-hand panel). Panel dimensions are $20 \times 20 \mathrm{arc} \mathrm{sec}^{2}$. The images have been smoothed with a gaussian kernel with radius $0 " .5$ (two pixels) for clarity. The compact narrow-band excess object on the top-left corner of the NB image is \#2620, one of the brightest fluorescent protogalactic or "dark" cloud candidates $\left(\mathrm{EW}_{0}>276 \AA\right.$ ) and undetected in both B and V-band).

simply because of the limit to the ratio of the number of ionizing photons to continuum photons at the Ly $\alpha$ wavelength from possible stellar populations. In the proximity of a quasar: the Ly $\alpha$ emission may be boosted by fluorescent emission, leaving untouched the stellar continuum, if present. The net effect is an EW "boost" of every object directly illuminated by the quasar by an amount that may vary with the object gas mass and its distance from the quasar. Therefore, if a significant fraction of our objects are indeed fluorescently boosted, our EW distribution should be skewed towards higher values with respect to surveys away from bright quasars.

Slightly different observational methods and selection techniques complicate the comparison to surveys in the literature. Despite these difficulties, the observed EW distri- butions from several recent 'blank-fields' surveys at $2.1<$ $z<2.8$ are remarkably similar. In particular, they can all be fitted by a single exponential function with an e-folding scale length of $w \simeq 50 \AA$. These surveys include: Grove et al. $2009\left(z=2.8, L_{l y \alpha}>3 \times 10^{41} \mathrm{erg} \mathrm{s}^{-1}\right.$; original data from Fynbo et al. 2004 ), Nilsson et al. $2009(z=2.3$, $\left.L_{l y \alpha}>2 \times 10^{42} \mathrm{erg} \mathrm{s}^{-1}\right)$, and Ciardullo et al. $2012(z=2.1$,

4 Extracted from a field containing a damped Ly $\alpha$ system at $z=2.85$ (Q2138-4427); we computed the EW distribution using all photometrically selected candidates in this field (Tables A3 and A4 in Grove et al. 2009). Note that, although this is not a strictly blank field since it contains a DLA, the over density of Ly $\alpha$ candidates is only a factor 1.1 with respect to the other surveys. 

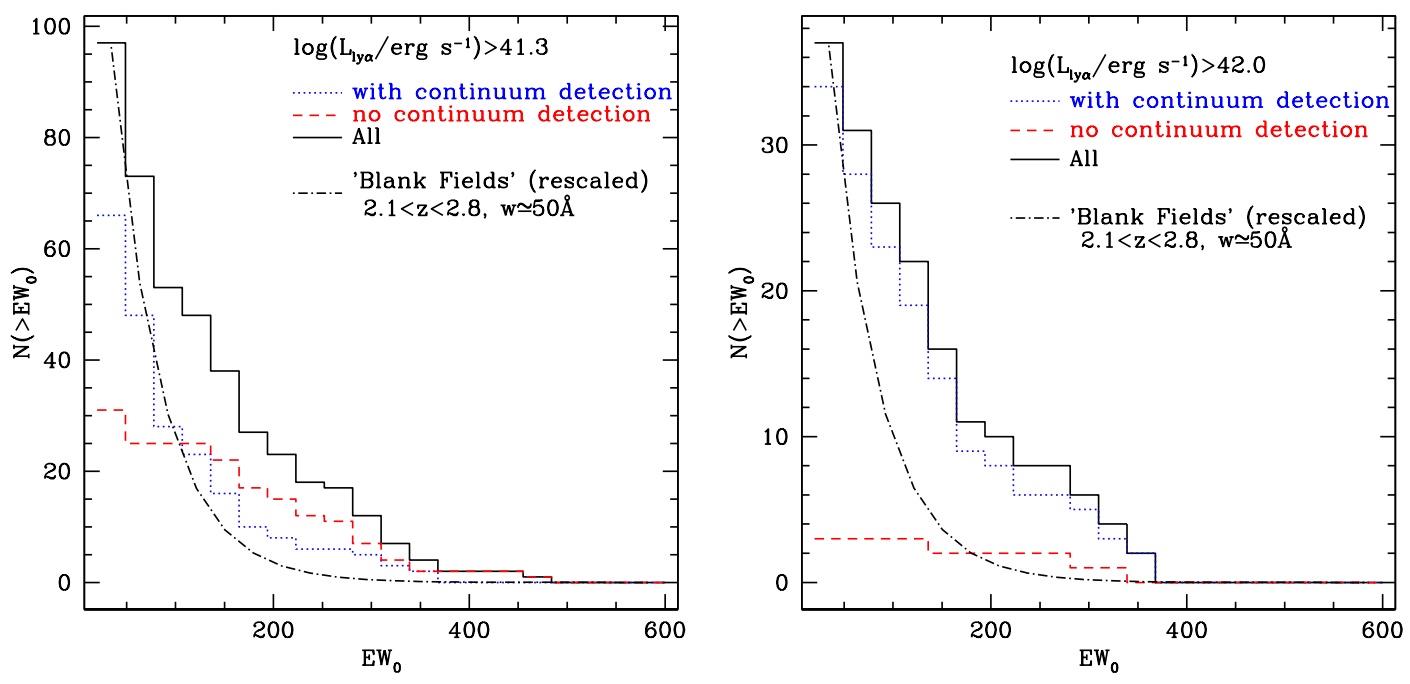

Figure 7. The cumulative rest frame equivalent width $\left(\mathrm{EW}_{0}\right)$ distribution for all the detected line emitting objects in our sample (lefthand panel; $L>2 \times 10^{41} \mathrm{erg} \mathrm{s}^{-1}$ ) and considering only the candidates with $L_{L y \alpha}>10^{42} \mathrm{erg} \mathrm{s}^{-1}$ (right-hand panel), always assuming the line is Ly $\alpha$. Objects are split according to their detection (blue dotted histogram) or non-detection (red dashed histogram) in any of the broad-bands. Note that the $\mathrm{EW}_{0}$ of the objects without continuum detections are lower limits. The solid black histogram shows the cumulative EW distribution including all objects and using the lower limits for the continuum-undetected candidates. The results from recent surveys targeting 'blank-fields', at $2.1<z<2.8$ are shown (black dot-dashed line, rescaled to match the total number of objects) taken from Ciardullo et al. $2012\left(z=2.1, L_{L y \alpha}>10^{42} \mathrm{erg} \mathrm{s}^{-1}\right.$; original data from Guaita et al. 2010), Nilsson et al. 2009 $\left(z=2.3, L_{L y \alpha}>2 \times 10^{42} \mathrm{erg} \mathrm{s}^{-1}\right)$, and Grove et al. $2009\left(z=2.8, L_{L y \alpha}>3 \times 10^{41} \mathrm{erg} \mathrm{s}^{-1}\right.$; original data from Fynbo et al. 2003$)$. The observed EW distributions from these surveys can all be fitted by a single exponential function with an e-folding scale length of $w \simeq 50 \AA$ (see text for details). Our sample presents a clear excess of high EW objects with respect to the 'blank-fields' surveys. This is true also considering only the continuum-detected objects or the more luminous part of the sample (right hand panel), This clear excess of high $\mathrm{EW}_{0}$ with respect to the field surveys is a distinctive sign of a fluorescent Ly $\alpha$ boosting due to the quasar proximity.

$L_{l y \alpha}>10^{42} \mathrm{erg} \mathrm{s}^{-1}$; original data from Guaita et al. 2010) 5 . As shown by these studies, the EW distribution seems to be also largely independent of the luminosity cut except at the very bright end, where there is an apparent lack of high EW objects. The EW distribution appears instead to evolve with redshift: at $z \sim 3.1$ there are evidences for an increase in $\mathrm{EW}$ scale length to $w \simeq 70 \AA$, (Ciardullo et al. 2012; Nilsson et al 2009; see also Ouchi et al. 2008) or larger at $z>4$ (e.g., Malhotra \& Rhoads 2002, Dawson et al. 2004, Saito et al. 2006, Shimasaku et al. 2006).

In Figure 7] we present the cumulative EW distribution for all the detected objects in our sample (left-hand panel; $L>2 \times 10^{41} \mathrm{erg} \mathrm{s}^{-1}$ ) and considering only the candidates with $L_{l y \alpha}>10^{42} \mathrm{erg} \mathrm{s}^{-1}$ (right-hand panel). The sample is divided according to whether the continuum was detected (blue dotted histogram) or not (red dashed histogram). The $\mathrm{EW}_{0}$ of the objects without continuum detections are of course therefore lower limits. The solid black histogram shows the cumulative EW distribution including all objects and using the lower limits for the continuumundetected candidates.

Our sample contains a clear excess of high EW objects with respect to the 'blank-field' surveys. This remains true also considering only the continuum-detected objects (that

5 The only exception is given by the results of Hayes et al. $(z=$ $2.2, L_{l y \alpha}>3 \times 10^{41}$ ) who find a slightly larger e-folding scale with value $w \simeq 76 \AA$ but they suggest that poor statistics are likely responsible for this discrepancy. show an e-folding scale of $w \simeq 85 \AA$ ) or when we consider only the most luminous part of the sample: in this case the e-folding scale for the continuum-detected objects (the large majority) is of the order of $w \simeq 120 \AA$, although the statistics are poorer. In both cases, a K-S test confirms that our EW distribution is different from the one obtained by the "blankfield" surveys at a significance greater than $99 \%$. It should be noted that we that we have not attempted to correct for the non-square bandpass of our filter since we have assumed that the Ly $\alpha$ emission of each candidate lies at the peak transmission wavelength of the filter (since we do not know the redshift distribution for all of our objects). We stress that this is a conservative assumption for the calculation of the EW and likely gives an underestimate of the true Ly $\alpha$ flux and EW of our candidates.

In addition to explaining the excess of high EW objects, the fluorescent Ly $\alpha$ boosting due to the proximity of the bright quasar is also able to account for the $w$ increase with luminosity that is exhibited by those objects with detected continua, the opposite trend to that exhibited by the "blank-field" surveys. Quasar fluorescence boosts both the Ly $\alpha$ luminosity and the EW of a given object (independent of its "original" position in the $\mathrm{L}_{l y \alpha}-\mathrm{EW}$ diagram ) creating a positive correlation between these two quantities.

Is there any other effect that could explain the different EW distribution obtained in our sample with respect to the "blank-field" surveys? Differently from the "blankfields", our sample could be associated with a high density peak in the matter distribution due to the presence of the 


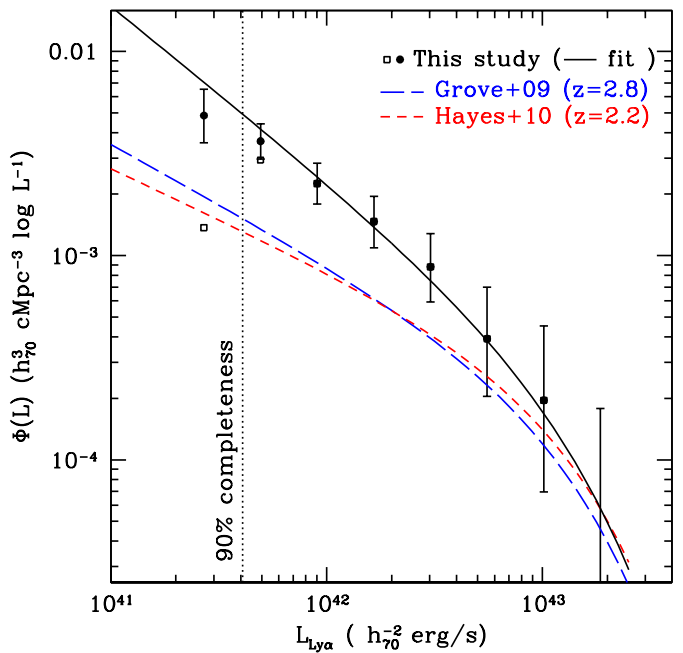

Figure 8. The luminosity function (number density per decade in luminosity) of our $\mathrm{Ly} \alpha$ candidates compared with other two deep surveys in "blank-fields" (see labels within figure). Solid circles (or open squares) show the observed Luminosity Function including (or excluding) the correction for completeness. The error bars show $84 \%$ confidence levels based on Poisson and binomial statistics (Gehrels 1986). Note that, consistently with the other surveys, we have not corrected for the non-square bandpass shape of the NB filter (see text for details). We detect a clear excess of sources at the faint end with respect to "blank-fields". As discussed in section 4.2 , this is fully compatible with fluorescent boosting due to quasar proximity.

quasar. We discuss extensively this possibility in the following sections. Venemans et al. 2007 performed a survey for Ly $\alpha$ emitters around high-redshift radio galaxies detecting a significant enhancement with respect to the field (about a factor 2-5). However, their EW distributions do not appear significantly different from the one obtained by the "blankfield" surveys. In particular, the fraction of candidates with $\mathrm{EW}_{0}>240 \AA$ (and $L_{l y \alpha}>10^{42} \mathrm{erg} \mathrm{s}^{-1}$ ) is less than 5\% (B. Venemans, private communication), similarly to the "blankfield" surveys at $2<z<3$. As a reference, we find instead that more than $20 \%$ of our sources with $L_{l y \alpha}>10^{42} \mathrm{erg} \mathrm{s}^{-1}$ have $\mathrm{EW}_{0}>240 \AA$.

\subsection{Luminosity Function}

Similarly to the EW distribution, we expect fluorescent boosting to also impact the Ly $\alpha$ Luminosity Function (LF) of our sample. In Figure 8, we show the LF obtained from all the Ly $\alpha$ candidates (open squares) and including a correction for completeness (solid circles; see section 2.3). We compare our LF with the results of Grove et al. 2009 and Hayes et al. 2010. These two surveys, the deepest available at $2<z<3$, reached a comparable depth and used a very similar NB filter on VLT-FORS with respect to our study. Despite the small volumes sampled, these two studies derived very similar luminosity functions, consistent with a Schechter function with $\log \left(L^{*} / \mathrm{erg} \mathrm{s}^{-1}\right) \sim 43.1$, slope $\alpha \sim-1.5$ and normalization $\log \left(\phi^{*} / \mathrm{cMpc}^{-3}\right) \sim-3.9$.

In our own survey, we detect a clear excess of Ly $\alpha$ can-
।

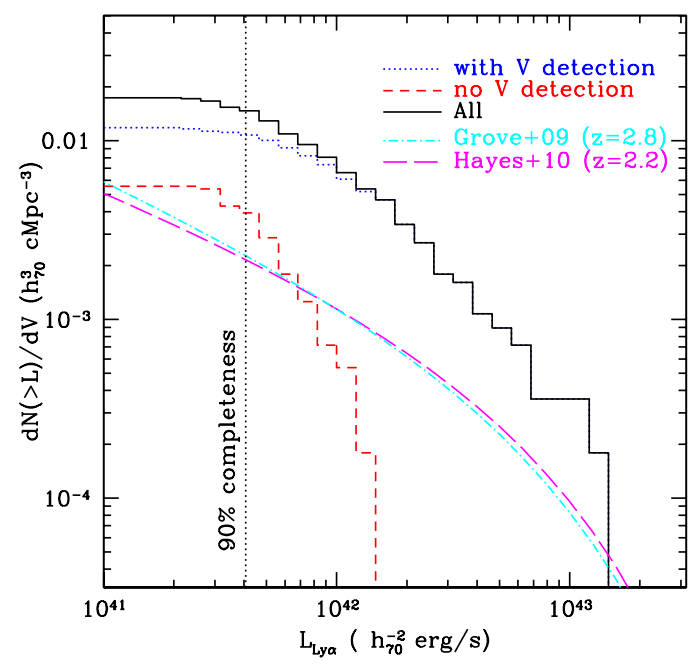

Figure 9. Cumulative luminosity function (CLF) of our Ly $\alpha$ candidates (black solid histogram) compared with other deep surveys in the field (see labels within figure). Blue dotted (or red dashed) histogram shows the CLF including only objects with (or without) continuum-detection.

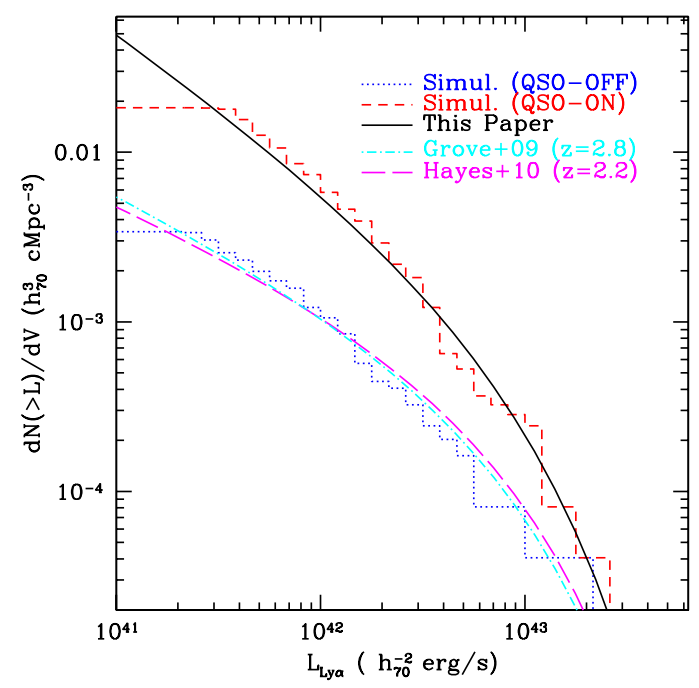

Figure 10. Simulated cumulative luminosity function (CLF) obtained from simulations assuming that the quasar is off (blue dotted histogram), i.e. no increase in the UV background due to quasar proximity, and that the quasar is turned on (red dashed histogram). These simulations include full three-dimensional radiative transfer of ionizing and Ly $\alpha$ photons (Cantalupo \& Porciani 2011; Cantalupo et al. 2005) within high-resolution cosmological simulations of structure formation around a massive halo (see text for details). The overall vertical normalization of the CLF of Ly $\alpha$ emitters in the QSO-OFF simulation has been rescaled to match the observed field CLF of deep surveys (Grove et al. 2009, Hayes et al. 2010), while the slope of the CLF is reproduced naturally by our simulation. Using the same normalization factor the numerical model is able to reproduce the increase in the total number of objects and the steepening of the CLF as observed in our data once the quasar is turned on. 
didates with respect to these "blank-fields". Fixing $L^{*}$ and the normalization to the same value of Grove et al. 2009 and Hayes et al. 2010 our data is best fit by a substantially steeper faint-end slope $(\alpha \simeq-1.9)$. As shown by the Cumulative Luminosity Function (CLF) in Figure 9 this excess of Ly $\alpha$ candidates is mostly driven by objects with continuum detections (blue dotted histogram), while the CLF for candidates without continuum detection (red dashed histogram) steeply rises only below $L<10^{42} \mathrm{erg} \mathrm{s}^{-1}$. The total number density above our $90 \%$ completeness limit (corresponding to $\left.L_{L y \alpha}>4 \times 10^{41} \mathrm{erg} \mathrm{s}^{-1}\right)$ is $n \sim 0.014 \mathrm{cMpc}^{-3}$, is about a factor 5 higher than Grove et al. 2009 and Hayes et al. 2010 at similar luminosities.

An enhancement in the number of Ly $\alpha$ emitters (LAE), i.e. in $\phi^{*}$ and possibly in $\mathrm{L}^{*}$, may be expected around a quasar simply because this is likely to occupy a high density region in the Universe. For instance, Venemans et al. (2007) found that LAEs around radio galaxies tend to be of a factor 2-5 more numerous with respect to the "field". A similar effect may be playing a role in our sample, although we note that radio-quiet quasars (like HE0109-3518) do not generally seem to show a different environment with respect to "normal", $L^{*}$ galaxies, at least on scales larger than 0.5 cMpc (e.g., Croom \& Shanks 1999, Serber et al. 2006, Falder et al. 2011; see also Hennawi et al. 2006). A very recent survey by Trainor \& Steidel (2012), obtained the same result also for hyper-luminous quasars (like HE0109-3518 ) at $z \sim 2.7$, concluding that the very low space density of such objects results from an extremely rare event on scales $<<1 \mathrm{cMpc}$ and not from being hosted by rare dark matter haloes.

Nevertheless, we also observe a steepening of the LF. As previously discussed, fluorescent boosting should enhance the Ly $\alpha$ emission of a gas rich object independent of its SFR (or any other combination of parameters that determines the Ly $\alpha$ luminosity of an isolated galaxy). This constant additive boost to the Ly $\alpha$ flux will have a larger relative effect at low luminosities, thereby steepening the faint end slope $\alpha$. This steepening is the clearest signature of fluorescent emission in the luminosity function.

In order to understand if the observed steepening of the LF is compatible with fluorescence, we performed a numerical experiment with the help of cosmological hydrodynamical simulations (using the hydro-code RAMSES, Teyssier 2002) combined with full three-dimensional radiative transfer for continuum (RADAMESH, Cantalupo \& Porciani 2011) and Ly $\alpha$ radiation (Cantalupo et al. 2005; Cantalupo et al. 2007). The simulations will be discussed in detail in a companion paper (Cantalupo et al., in preparation). In Appendix B, we briefly describe the numerical methods and simulation parameters.

The simulations have been used to produce "mock" observations of Ly $\alpha$ emitting objects in a $40^{3} \mathrm{cMpc}$ volume of the Universe at $z=2.4$ around a quasar. The latter has been turned on and off in order to simulate the differential effect of fluorescent boosting on the LF. The results are presented in Fig. 10. In the "QSO-OFF" simulation we have assigned a Ly $\alpha$ emissivity to each object proportionally to its SFR (see Appendix B) plus the recombination radiation due to the UVB background ionizations, i.e. UVB fluorescence (several orders of magnitude below our detection threshold). Then, we applied our Ly $\alpha$ RT, added the observed noise to the simulated image and selected candidates using the same SExtractor procedure applied to the real data. The LF obtained in this way (blue histogram) naturally reproduces the observed shape of the LF in "blank-fields" once a normalization factor $n_{\mathrm{ly} \alpha}=0.13$ is applied to take into account the fraction of galaxies appearing as Ly $\alpha$ emitters and the overdensity of our simulation box (see Appendix B). We then turned on a quasar in the most massive halo in the simulation (located at its center) and propagated its ionizing photons through hydrogen and helium within the box using our radiative transfer code RADAMESH. We then repeated exactly the procedure followed for the "QSO-OFF" simulation, using the same parameters and normalization factor of the latter. Thus the only difference between the two simulations is the presence of the quasar ionization field. The resulting $\mathrm{LF}$ in the "QSO-ON" case is shown as a red histogram in Figure 10. The steepening in the simulated LFs is clearly visible and is very similar to the difference between the observed LF in the "blank-field" surveys and in our sample around the quasar. Within the limitation of numerical simulations and observational errors, this provides an additional support for the idea that fluorescence is playing a major role in our sample

\subsection{Luminosity-Distance Relation}

An important prediction for quasar fluorescence is the relation for optically thick clouds between the fluorescent Surface Brightness (SB) and the (three-dimensional) distance from the quasar (e.g., Cantalupo et al. 2005). This is because the fluorescent boost is proportional to the apparent brightness of the quasar as seen by the cloud. Optically thick objects whose size is much larger than the ionized region act like a "mirror" of the impinging quasar radiation (Gould \& Weinberg 1996). Their fluorescent SB would thus simply scale like the inverse square of their three-dimensional distance from the quasar, assuming isotropic and temporally constant quasar emission. In reality, however, geometrical and kinematical effects can reduce the expected SB by a large factor, although this reduction can be parametrized as shown in Cantalupo et al. (2005).

Furthermore, the SB of those gas clouds that have been completely ionized by the quasar will be independent of distance from the quasar (for as long as they are completely ionized). Their luminosity is simply proportional to the integral of their hydrogen density squared (see section [5.2). Finally, any local star formation might add a substantial Ly $\alpha$ flux to the fluorescent component, and this also would be expected to be independent of distance to the quasar.

Despite these caveats, if a significant fraction of optically thick clouds ("fluorescent mirrors") are present, we might still expect to find an increase of the average Ly $\alpha$ $\mathrm{SB}$ in our sample closer to the quasar. Unfortunately, we cannot obtain directly either the surface brightness or the three-dimensional distance from the quasar for most of our objects. This is due to their compact nature and the lack, so far, of spectroscopic data for the whole sample (a spectroscopic follow-up of the brightest objects will be presented in Cantalupo et al., in prep.). However, we can use the total Ly $\alpha$ luminosity and the projected distance from the quasar as proxies for these two quantities, as shown for our sample in Fig. 11, Although the statistics are poor, we do find 


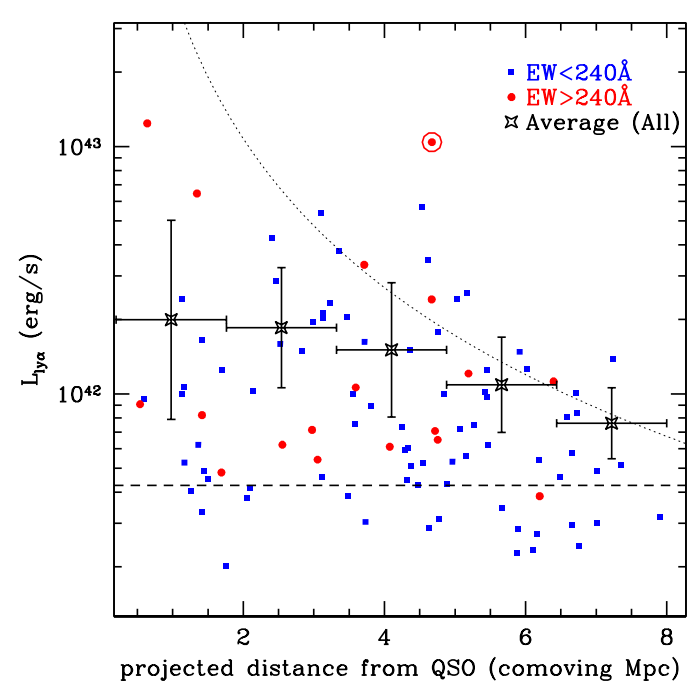

Figure 11. The luminosity of Ly $\alpha$ candidates as a function of projected distance from the quasar. The dotted line shows the expected fluorescent $\mathrm{Ly} \alpha$ emission produced by an optically thick cloud of $1 \operatorname{arcsec}^{2}$ area as a function of its three-dimensional distance from the quasar. This represents an upper limit for fluorescent protogalactic clouds without star formation at the quasar redshift. The luminosity would scale linearly with object area, but the majority of detected objects are compact. One notable exception is \#1159 (circled), that has an area of $\sim 20 \mathrm{arcsec}^{2}$. The dashed line represents the $90 \%$ completeness limit of the survey. We have used all the objects above this limit (except the very extended \#1159) to compute the average luminosity per projected distance bin (starred open squares). If a significant number of optically thick clouds are present and the objects have Ly $\alpha$ emission boosted by quasar fluorescence, then we expect the average luminosity to decrease approximatively with the square of the distance from the quasar (assuming no intrinsic relation between Ly $\alpha$ emission from star formation and distance from the quasar). Despite the large errorbars, there is a marginal detection of the expected signal. This is also confirmed by the results of our radiative transfer simulations presented in figure 12

indeed that the average luminosity above the completeness level decreases with the projected distance from the quasar (starred open squares).

As a reference, we have plotted on the same figure the expected "mirror" fluorescent Ly $\alpha$ luminosity of a optically thick cloud with an area of $1 \operatorname{arcsec}^{2}$ as a function of threedimensional distance from the quasar (dotted line). This will represent the "maximum" emission for a purely fluorescent source of this area (e.g. Cantalupo et al. 2005). Although the uncertainties related to projection effects and object sizes are quite large, it is interesting to note that the expected trends are similar to those observed, except in the close proximity of the quasar. This is the region where we would expect the quasar radiation to be so intense that very few clouds would be able to remain optically thick.

A better analysis that included the size, projection and ionization effects can be performed with the help of the radiative transfer simulations presented in the previous section and discussed in detail in Appendix B. In Fig. 12 we show the differential effect of fluorescent boosting on the simulated projected distance-luminosity relation once the quasar

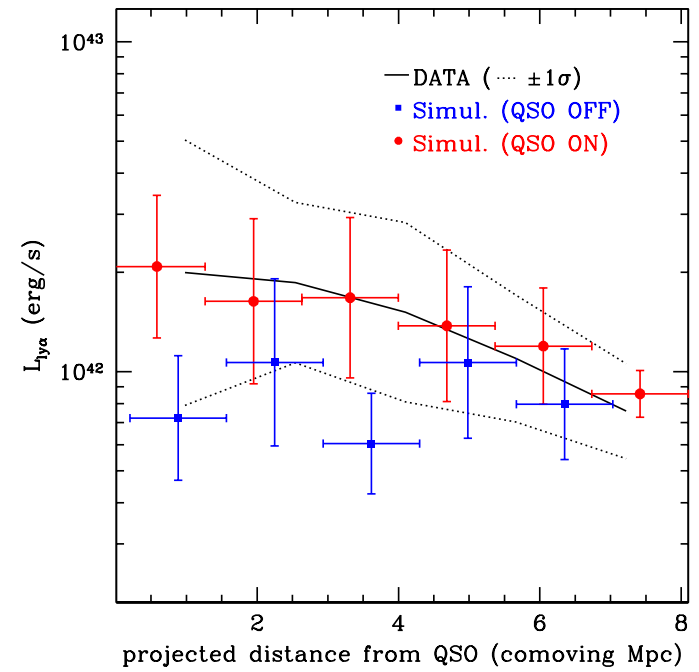

Figure 12. Comparison between the average luminosity of observed Ly $\alpha$ candidates (black solid line) and the average luminosity of the simulated Ly $\alpha$ emitters (blue squares for QSO-OFF and red dots for QSO-ON simulations) as a function of projected distance from the quasar above the same luminosity cut as in figure 11 Despite large errorbars, the observed trend is very similar to the QSO-ON simulation suggesting that the observed signal is compatible with fluorescent emission boosted by the quasar proximity.

is turned-on. The trend obtained from the QSO-ON simulations (red dots) is now in much better agreement with the observations (solid line) at all radii. Note that this trend was not present without the effect of the quasar radiation (blue dots). Within the limitations of our numerical models, this is a further support that fluorescent boosting is playing a significant role in the $\mathrm{Ly} \alpha$ emission of the objects detected in our survey.

\section{THE NATURE OF THE SOURCES}

The statistical analyses performed in the previous section indicate that the dominant emission mechanism for many of our sources is likely to be Ly $\alpha$ fluorescence induced by the quasar. This mechanism then gives us a unique opportunity to directly image, in emission, dense gas independent of any associated star formation. This feature distinguishes these new observations from previous surveys for Ly $\alpha$ emitting galaxies or other structures. Moreover, fluorescent boosting increases the Ly $\alpha$ luminosity of every galaxy, if illuminated by the quasar that is gas rich. In principle, this makes intrinsically faint Ly $\alpha$ emitters, that would have been below our detection threshold, visible in our survey. This raises the obvious question: what are the nature and physical properties of this new population of sources and how do they differ from previously discovered Ly $\alpha$ emitters?

\subsection{Proto-galactic clouds and faint galaxies}

As previously discussed, a high Ly $\alpha$ EW (e.g., $\mathrm{EW}_{0}>240 \AA$ ) is the first criterion to exclude objects in which Ly $\alpha$ emis- 
sion is powered (solely) by star-formation. In our sample of 98 narrow-band excess objects, we detected 18 sources with equivalent width measurements or lower limits above $\mathrm{EW}_{0}>240 \AA$

Among these 18 objects with demonstrated $\mathrm{EW}_{0}>$ $240 \AA$, all but six do not have any detectable continuum counterpart and therefore quasar fluorescence is likely the dominant source of $\operatorname{Ly} \alpha$ photons. Their Ly $\alpha$ luminosities, which are all around or below $10^{42} \mathrm{erg} \mathrm{s}^{-1}$ (see Figure 9) are also fully compatible with fluorescence given their distance from the quasar and their compact sizes, as shown in Figure 11.

It should be noted in passing that a further 19 objects do not have any continuum detection and could also be entirely powered by quasar fluorescence. However, the broadband images are not deep enough to require their $\mathrm{EW}_{0}$ to lie above the $240 \AA$ level.

It is clear from Figure 9 that the steepening of the cumulative Luminosity Function with respect to the "blanksurveys" involves both objects with and without continuum detections - it does not come from the non-continuum detected objects alone, and is mostly due to the larger number of continuum-detected objects that are also presumably boosted by quasar fluorescence. The existence of these fluorescently boosted continuum-detected galaxies is confirmed by the EW distributions presented in Figure 7 especially when we restrict to the bright sample with $\mathrm{L}_{l y \alpha}>10^{42}$ erg $\mathrm{s}^{-1}$. As discussed in section 4.2 our radiative transfer simulations naturally reproduce the steepening of the cumulative luminosity function (see Figure 10) and the variation of the average Ly $\alpha$ luminosity with distance from the quasar (see Figure 12) once quasar radiation is "turned-on". It is therefore instructive to have a closer look at the physical properties of the simulated sources.

In Figure 13 we show the relation between the SFR and Ly $\alpha$ luminosity of the simulated emitters. This has been obtained performing SExtractor aperture photometry (after adding artificial noise to the image) in the same way as with the actual data. In the "QSO-OFF" simulation, the only "source" of Ly $\alpha$ photons are the stars. The intrinsic Ly $\alpha$ emissivity is set proportional to the SFR (see appendix $\mathrm{B}$ for details). In the absence of radiative transfer effects the simulated objects in the "QSO-OFF" simulation (blue open squares) would thus lie on the relation indicated by the black dotted line. It should be noted that our simulations do not include absorption by dust particles, and the reduction in the recovered luminosity of $\operatorname{Ly} \alpha$ emitting galaxies is due to aperture photometry losses. A significant fraction of Ly $\alpha$ photons are scattered by the circumgalactic and Intergalactic medium, forming a diffuse low surface brightness halo. This radiative transfer effect was discussed in detail in Cantalupo et al. 2005 (see also, e.g., Zheng et al. 2011, Barnes et al. 2011). On average, SExtractor aperture photometry only recovers about $20 \%$ of the total luminosity. This is in agreement with the recent observational estimate of Steidel et al. (2011) from a stacking analysis. Once the quasar radiation is turned on, in the simulation, the Ly $\alpha$ luminosities change, as shown by the solid red dots. Clearly, fluorescent boosting makes the simulated galaxies "overluminous" with respect to their SFR, increasing the number of detectable objects. This is due both to the increased emissivity from recombination radiation and, to a lesser extent, to the reduced

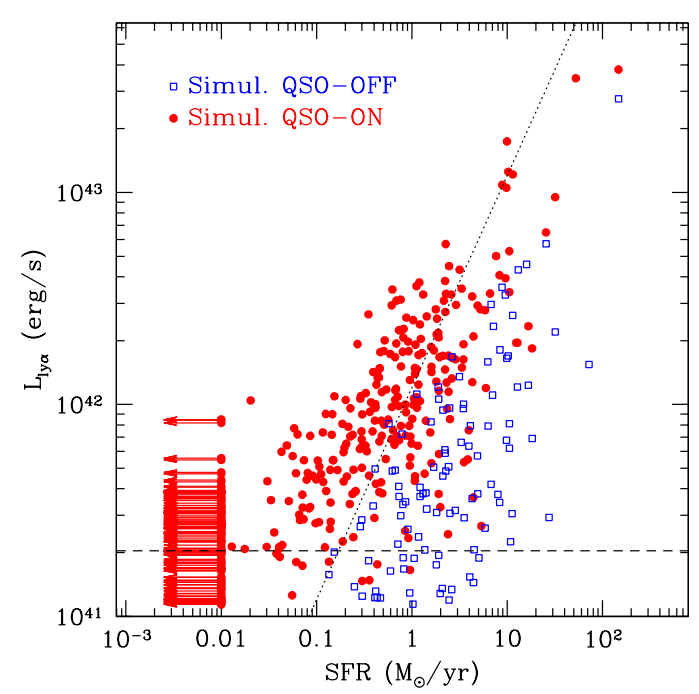

Figure 13. Luminosity versus SFR for the $\operatorname{Ly} \alpha$ emitters in the QSO-OFF (blue squares) and QSO-ON (red circles) simulations. In the QSO-OFF simulations, the intrinsic Ly $\alpha$ emissivity is set proportional to the SFR ( see Appendix B for details). After adding simulated noise, the object luminosities are obtained with SExactor aperture photometry, similarly to the treatment of the actual data. Note that the simulations include the full threedimensional scattering of $\operatorname{Ly} \alpha$ photons within the circumgalactic and intergalactic medium (Cantalupo et al. 2005) but they do not include dust absorption. Without radiative transfer effects, the Ly $\alpha$ emitters in the QSO-OFF simulation would therefore lie on the intrinsic luminosity-SFR relation given by the dotted black line, which represents a "maximum" value for the recovered Ly $\alpha$ emission powered by star formation. Quasar fluorescence in the QSO-ON simulation (red dots) boosts many objects above this limit, especially at lower SFRs, generating the steepening of the CLF observed in Figure 10 About $20 \%$ of sources in the simulation lying above our nominal detection threshold (dashed line) are not associated with star-formation within the simulation (at least above our numerical resolution limit of $\left.\sim 0.01 M_{\odot} \mathrm{yr}^{-1}\right)$. They are represented by left-pointing arrows at $\mathrm{SFR}=0.01$. Their relative abundances and luminosities are very similar to the observed objects without continuum detection in our survey strengthening the association between the detected continuum-less sources and proto-galactic clouds.

Ly $\alpha$ scattering in the more ionized circum-galactic medium. In particular, the simulation suggests that the steepening of the observed luminosity function is due to galaxies that would otherwise be one order of magnitude fainter in Ly $\alpha$. Our survey strategy gives thus the possibility to select and study intrinsically faint galaxies at high-redshift at an incomparably deep level. This is similar in some respects to the use of gravitational lenses, although in our case only the Ly $\alpha$ emission is "boosted". In particular, we should have reached similar levels to the ultra-deep blind Ly $\alpha$ spectroscopic search of Rauch et al. 2008, although a direct comparison is complicated by the very different observational techniques.

An interesting point is that in the "QSO-ON" simulation, we see sources (the red dots with arrows) that do not have any associated star formation at the simulation resolution limit for star-formation of about $0.01 \mathrm{M}_{\odot} \mathrm{yr}^{-1}$. 


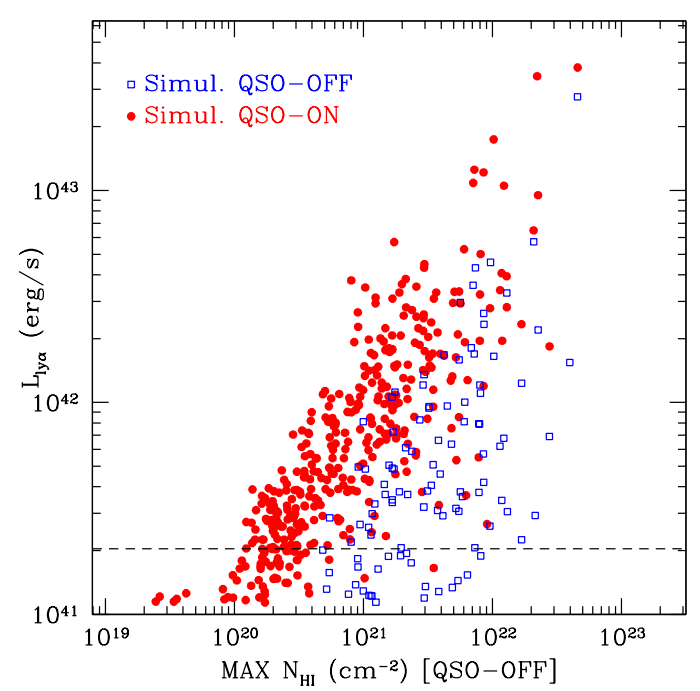

Figure 14. The relation in the simulations between the Ly $\alpha$ luminosity and the maximum HI column density before the quasar is turned on (see labels within the panel). The maximum $\mathrm{N}_{\mathrm{HI}}$ is defined considering all the lines-of-sight that intersect any image pixel with detectable Ly $\alpha$ emission. Note that we do not simulate the formation of $\mathrm{H}_{2}$, and thus the reported $\mathrm{HI}$ values will be an overestimate, especially at $\mathrm{N}_{\mathrm{HI}}>10^{21} \mathrm{~cm}^{-2}$.

These objects are all below a luminosity of about $10^{42} \mathrm{erg}$ $\mathrm{s}^{-1}$ and represent about $20 \%$ of the total number of sources above our observational threshold. Their luminosities and relative abundances are remarkably similar to the observed objects that did not have continuum-detection. A closer look at these simulated sources reveals that they are compact dense clouds, proto-galaxies in the transition phase between the IGM and ISM, with peak densities that are not yet large enough to currently produce and sustain significant star formation 6 .

Typically located within dense knots in IGM filaments, these clouds will eventually increase their gas density because of cosmological gas infall and start to form stars. Such a "proto-galactic phase" for gas rich and dense clouds might recently have been detected from the study of high column density absorption systems such as LLSs (Fumagalli, O'Meara \& Prochaska 2011) and among metal-poor DLAs (Penprase et al. 2010; Cooke et al. 2011; Cooke, Pettini \& Murphy 2012; but see Carswell et al. 2012). Unfortunately, the lack of spatial and morphological information intrinsic to these one-dimensional absorption studies does not allow us to determine whether these clouds are truly isolated systems or part of larger galactic gas reservoirs, either circum-galactic (e.g., streams-like features) or within galaxies. Given the statistical evidences for fluorescence in our sample and the support from simulations, our data provides - in emission - strong indications for the existence of such compact and isolated proto-galactic or "dark" clouds,

6 From a numerical point of view, this means that their peak density was always below the assumed star formation threshold in the simulations of $n_{\mathrm{ISM}}=1$ atoms $\mathrm{cm}^{-3}$. opening up a new observational window for the study of the early phases of galaxy formation.

How are these emitters then related to QSO absorption line systems? In Figure 14 we show the relation between the Ly $\alpha$ luminosity of our simulated sources and the maximum HI column density before the quasar is turned on. The latter is defined as the maximum value among the line-ofsight that intersects any pixel with detectable Ly $\alpha$ emission. We do not simulate the formation of molecular hydrogen, therefore the reported HI values are likely to be an overestimate, especially at $\mathrm{N}_{\mathrm{HI}}>10^{21} \mathrm{~cm}^{-2}$. As expected, Ly $\alpha$ emitting galaxies in the QSO-OFF simulation are only associated with the highest $\mathrm{N}_{\mathrm{HI}}$ systems, i.e, $\mathrm{N}_{\mathrm{HI}}>5 \times 10^{20}$ $\mathrm{cm}^{-2}$, above the DLA threshold $\left(2 \times 10^{20} \mathrm{~cm}^{-2}\right)$. This is in a sense by construction, since we only allowed star-formation to take place above a given density threshold $\left(1\right.$ atom $\left.\mathrm{cm}^{-3}\right)$ following the Schmidt-Kennicutt law (see Appendix B). Indeed, this density threshold translates into a critical column density (given by the Toomre instability criterion ) that in local galaxies is observed to range between $5 \times 10^{20} \mathrm{~cm}^{-2}$ and $2 \times 10^{21} \mathrm{~cm}^{-2}$ (Kennicutt 1998).

Once the quasar is turned on, we notice that the overall boost in luminosity still seems to correlate with the initial HI column density of the quasar-off simulation. This is because higher column density systems are typically larger, more massive and denser. The proto-galactic clouds without star-formation start to appear at values of $\mathrm{N}_{\mathrm{HI}}$ around or below the simulation critical SF threshold $\left(5 \times 10^{20} \mathrm{~cm}^{-2}\right)$. In particular, the simulation suggests that the objects fluorescently illuminated by the quasar have typical $\mathrm{N}_{\mathrm{HI}}$ - when the quasar is off - one order of magnitude lower than the sources detectable by similarly deep "blank-field" surveys. Given our current sensitivity limits, we are able to detect only the high-end tail of the $\mathrm{N}_{\mathrm{HI}}$ distribution associated with these clouds. Deeper surveys, e.g. with integral field spectrographs, could reveal many more such systems.

\subsection{The gas mass and star formation efficiency of protogalactic haloes}

If we can assume that there is negligible Ly $\alpha$ emission produced by internal star formation, then we can directly convert the Ly $\alpha$ luminosity produced by HII recombination into an estimate of the total HII mass. Indeed, in the absence of dust absorption and HI collisional excitations (in other words, if the gas is mostly ionized), then the total Ly $\alpha$ luminosity is simply given by (e.g., Cantalupo et al. 2008):

$$
L_{\mathrm{ly} \alpha}=\int_{V} n_{\mathrm{e}} \cdot n_{\mathrm{HII}} \cdot \alpha_{\mathrm{ly} \alpha}^{\mathrm{eff}}(T) h \nu_{\mathrm{ly} \alpha} \mathrm{d} V
$$

where $n_{\mathrm{e}}, n_{\mathrm{HII}}, \alpha_{\mathrm{ly} \alpha}^{\mathrm{eff}}(T)$ and $h \nu_{\mathrm{ly} \alpha}$ are, respectively, the electron number density, the proton number density, the effective Ly $\alpha$ emission coefficient (see Cantalupo et al. 2008), and the Ly $\alpha$ photon energy. Assuming a uniform temperature and ionization fraction within the cloud, we can rewrite eq2 as:

$$
L_{\mathrm{ly} \alpha}=\chi_{\mathrm{e}} \alpha_{\mathrm{ly} \alpha}^{\mathrm{eff}}(T) h \nu_{\mathrm{ly} \alpha} \int_{V} n_{\mathrm{HII}}^{2} \mathrm{~d} V
$$




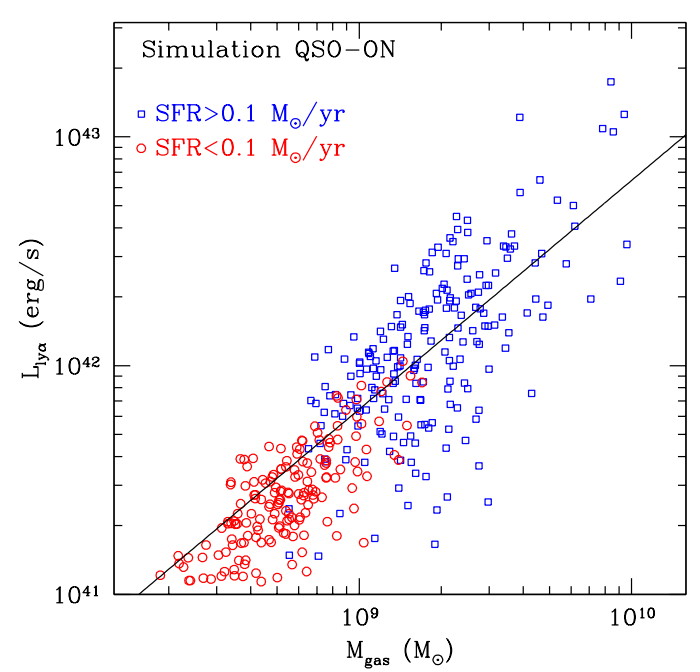

Figure 15. The relation between the cloud gas mass and Ly $\alpha$ luminosity within our simulation with the quasar turned on. We have included only gas with $T<5 \times 10^{4} \mathrm{~K}$. The solid line is the analytical expected relation (eq 8 ) for fully ionized objects without star formation, derived from the halo cosmological scaling laws and assuming a fixed gas temperature (see text for details). This relation gives a good approximation for the expected mass-luminosity function of fluorescently illuminated gas clouds with absent or little star-formation, as derived from our radiative transfer simulation. Both analytical arguments and the numerical simulation suggest therefore that the gas mass of the detected protogalactic candidates would, if it is mostly ionized, be of the order of $10^{9} \mathrm{M}_{\odot}$ or larger.

where, $\chi_{\mathrm{e}}$ is the electron fraction $(\sim 1.16$ if $\mathrm{H}$ and $\mathrm{He}$ are fully ionized). Using the definition of clumping factor:

$$
C=\frac{<n_{\mathrm{HII}}^{2}>}{\left.<n_{\mathrm{HII}}\right\rangle^{2}}=\frac{\int_{V} n_{\mathrm{HII}}^{2} \mathrm{~d} V}{\left[\int_{V} n_{\mathrm{HII}} \mathrm{d} V\right]^{2}} \times V,
$$

we thus obtain:

$$
L_{\mathrm{ly} \alpha}=\chi_{\mathrm{e}} \alpha_{\mathrm{ly} \alpha}^{\mathrm{eff}}(T) h \nu_{\mathrm{ly} \alpha} \cdot C \cdot V^{-1} M_{\mathrm{HII}}^{2} .
$$

For a spherical, fully ionized cloud with radius $R$, gas mass $M_{\text {gas }}$ and fixed temperature in the range $10^{4} \mathrm{~K}<T<$ $10^{4.7} \mathrm{~K}$, eq 5 becomes:

$L_{\mathrm{ly} \alpha} \sim 1.4 \times 10^{43} \mathrm{erg} / \mathrm{s} \cdot C \cdot\left[\frac{2 \cdot 10^{4} \mathrm{~K}}{T}\right]\left[\frac{R}{\mathrm{pkpc}}\right]^{-3}\left[\frac{M_{\mathrm{gas}}}{10^{9} M_{\odot}}\right]^{2}$,

where pkpc denotes physical kpc. With the knowledge of the cloud size we could therefore derive its gas mass from the Ly $\alpha$ luminosity.

Unfortunately, our proto-galactic candidates are essentially unresolved in our ground-based image. In absence of this information, we can assume that the gas is settled into a disk at the center of its dark matter halo and derive a proxy for the object size from the cosmological scaling relations between virial radius and mass (e.g., Mo, Mao \& White 1998). In particular, assuming that the disk size and gas mass are fixed fractions (respectively, $f_{r}$ and $f_{g}$ ) of the dark-matter halo virial radius and total mass, we have (at $z=2.4$ ):

$$
R \sim 2.7 \mathrm{pkpc}\left[\frac{M_{\mathrm{gas}}}{10^{9} M_{\odot}}\right]^{1 / 3}\left[\frac{f_{g}}{0.05}\right]^{-1 / 3}\left[\frac{f_{r}}{0.1}\right],
$$

where we have used the same values of the disk to total mass ratio $\left(f_{g}\right)$ and disk to virial radius ratio $\left(f_{r}\right)$ as in Mo et al. (1998). It should be noted for consistency that eq[7 implies that a disk with $M_{\text {gas }} \sim 10^{9} M_{\odot}$ would have a size of less than 0.7" at this redshift and therefore it would appear unresolved in our image. Using eq[7 as a proxy for the cloud size (and dropping the dependency on $f_{g}$ and $f_{r}$ for simplicity) we can thus rewrite eq 6 as:

$$
M_{\text {gas }} \sim 1.4 \times 10^{9} M_{\odot}\left[\frac{L_{\mathrm{ly} \alpha}}{10^{42} \mathrm{erg} \mathrm{s}^{-1}}\right]\left[\frac{T}{2 \times 10^{4} K}\right] \cdot C^{-1} .
$$

We stress that the mass derived with this approach is representative of the cloud gas mass only for systems that are highly ionized by the quasar radiation and with a given temperature. In the other cases, eq 8 underestimates the value of $M_{\text {gas }}$, unless there is a significant Ly $\alpha$ emission contribution from HI collisional excitation, e.g. from cooling. However, highly ionized, optically thick objects may be distinguished from cooling clouds using the Ly $\alpha$ spectral and SB profile (see, e.g. Cantalupo et al. 2005, Dijkstra et al. 2006, Cantalupo et al. 2007) or with the help of other diagnostic

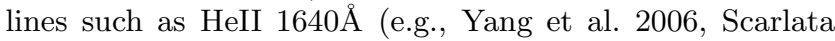
et al. 2009). Future spectroscopic follow-up and space-based imaging of our sources may therefore help to understand if eq 8 is applicabile in these objects. In the current absence of this information, however, we can use our radiative transfer simulation to have an idea of the expected mass-luminosity relation for a variety of objects with different ionization fractions and temperature distributions.

In Fig. 15] we show the relation between the cloud gas mass and its Ly $\alpha$ luminosity in our radiative transfer simulations, once the quasar is turned on. We have considered only gas with $T<5 \times 10^{4} \mathrm{~K}$ - the main component producing Ly $\alpha$ photons from recombination - and lying within the aperture radius given by SExtractor photometry. We divide the sample according to the SFR of the object. For clouds with $\mathrm{SFR}<0.1 M_{\odot} \mathrm{yr}^{-1}$ (red circles), the Ly $\alpha$ luminosity is mostly powered by quasar radiation. These systems appear to follow well the analytical trend with mass. As expected, eq 8 tends to underestimate the total gas mass, although we note that a higher temperature than $2 \times 10^{4} \mathrm{~K}$ would help to recover the value obtained in the simulations.

From eq 8 and with the help of the radiative transfer simulation, we can therefore derive an estimate of the gas mass of those Ly $\alpha$ sources without continuum detection and with $\mathrm{EW}_{0}>240 \AA$. Their typical Ly $\alpha$ luminosity are in the range $0.5-1 \times 10^{42} \mathrm{erg} \mathrm{s}^{-1}$ implying therefore a gas mass of around $M_{\text {gas }} \sim 10^{9} M_{\odot}$, or higher if they are not mostly ionized by the quasar radiation. We can derive a constraint on the average SFR of these systems from the non-detection in the V-band stack (see Fig 4) at the level of $V(1 \sigma) \sim 30.3$. We convert this observed magnitude limit (corresponding to rest-frame wavelength of about $1600 \AA$ ) into a SFR upper limit using Starburst99 (Leitherer et al. 1999) and Oti-Floranes \& Mas-Hesse (2010). Assuming an extended burst of duration 250Myr, Salpeter IMF (1-100 $\left.M_{\odot}\right)$ and $E(B-V)=0$ we obtain a $\mathrm{SFR}<10^{-2} M_{\odot} \mathrm{yr}^{-1}$. This implies a Star Formation Efficiency (SFE) for these objects of $\mathrm{SFE} \equiv \mathrm{SFR} / M_{\text {gas }}<10^{-11} \mathrm{yr}^{-1}$.

This very low SFE is several times lower than the typical SFE of dwarf galaxies in the local Universe (e.g., Geha et al. 2006). It is also 200 times lower than the SFE of typ- 


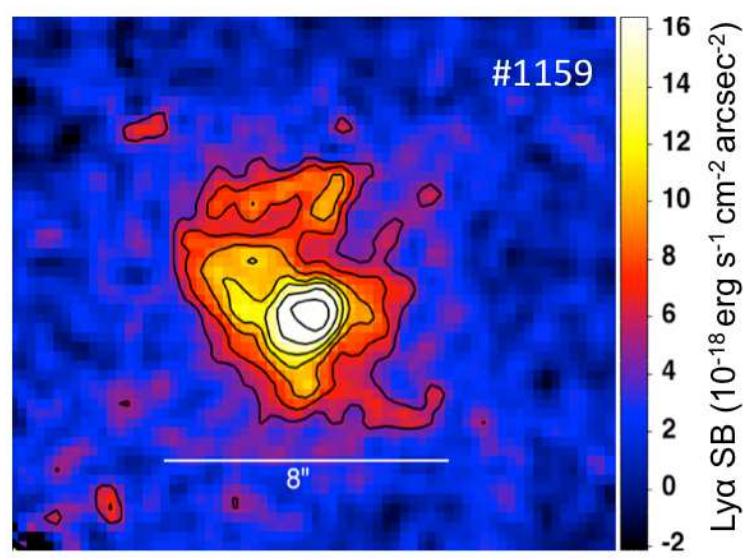

Figure 16. Continuum subtracted NB image of \#1159 revealing the extended, filamentary emission surrounding the central source. Contours show isophotal surfaces ( $1 \sigma$ intervals, starting from $2 \sigma$ ). Size and luminosity of this emission are compatible with the circumgalactic medium illuminated by the quasar (see text for details). Note that 8" corresponds to about 66 physical $\mathrm{kpc}$ at $\mathrm{z}=2.4$, i.e. the virial diameter of a halo with total mass $M \sim 4 \times 10^{11} M_{\odot}$.

ical main sequence galaxies at $z \sim 2$ which has been estimated to be around SFE $\sim 2 \times 10^{-9} \mathrm{yr}^{-1}$ (Daddi et al. 2010, Genzel et al. 2010). Although we currently have only upper limits on the cloud sizes, it is interesting to estimate where our sources would lay with respect to the KennicuttSchmidt (KS) relation (Kennicutt 1998) between SFR per unit area $\left(\Sigma_{\text {SFR }}\right)$ and gas surface density $\left(\Sigma_{\text {gas }}\right)$. From the estimated gas mass $M_{\text {gas }} \sim 10^{9} M_{\odot}$, the SFR upper limit and assuming that our objects follow the cosmological scaling relation between mass and radius (eq. 7), we would obtain an average $\Sigma_{\text {gas }} \sim 44 M_{\odot} \mathrm{pc}^{-2}$ and $\Sigma_{\mathrm{SFR}}<4 \times 10^{-4} M_{\odot}$ $\mathrm{yr}^{-1} \mathrm{Kpc}^{-2}$. This value of $\Sigma_{\mathrm{SFR}}$ is about 100 times lower than the expected value from the local $\mathrm{KS}$ relation but it is consistent with the metallicity-dependent models of Krumholz, McKee and Tumlinson (2009) and Gnedin \& Kravtsov (2011) that predict a substantial steepening of the $\mathrm{KS}$ relation at $\Sigma_{\text {gas }} \sim 50-100 M_{\odot}$ for very low metallicity gas $\left(Z<0.01 Z_{\odot}\right)$ at high redshift (see also Rafelski, Wolfe $\&$ Chen 2011).

\subsection{Cold Circum-Galactic Medium}

A different category of objects is represented by the more luminous, continuum-detected sources with EW> $240 \AA$, which show evidence of extended emission (left-hand panels in Figure 5). These are among the brightest Ly $\alpha$ sources in our sample and therefore it is not surprising that we detected some stellar continuum associated with them. Their extended emission has a surface brightness at a level of $0.5-1 \times 10^{-17} \mathrm{erg} \mathrm{s}^{-1} \mathrm{~cm}^{-2} \operatorname{arcsec}^{-2}$, a value compatible with the quasar induced fluorescence suggested by the high EW. Their luminosities and low number density suggests, on the basis of our simulations, an association with the most massive systems in our surveyed volume, i.e. with objects with halo masses between $10^{11}$ and $10^{12} M_{\odot}$. Several theoretical and numerical studies have suggested that these haloes are able to retain dense and cold gas reservoirs in the form of filaments in the circum-galactic medium (e.g., Dekel \& Birnboim 2006, Ocvirk \& Teyssier 2008, Dekel et al. 2009, Keres et al. 2009, Fumagalli et al. 2011, van de Voort \& Schaye 2012). At the densities and temperatures predicted by these models, these circum-galactic filaments could be detectable in fluorescent emission in the proximity of a bright quasar. Note that this is a different mechanism with respect to Ly $\alpha$ cooling emission - suggested as a possible origin for extended Ly $\alpha$ structures like Ly $\alpha$ blobs, away from bright quasars (e.g., Haiman, Spaans \& Quataert 2000, Fardal et al. 2001, Dijkstra \& Loeb 2009, Goerdt et al. 2010; but see Furlanetto et al. 2005, Faucher-Giguere et al. 2010; Rosdahl \& Blaizot 2011). Mostly due to HII recombinations, quasar fluorescent emission is much less sensitive to the gas temperature state (see e.g., Fig. 1 in Cantalupo et al. 2008) and therefore it is much easier to estimate the expected Ly $\alpha$ surface brightness (see Appendix B for further discussion). It is intriguing that one of the brightest objects in our sample, \#1159, indeed shows some evidence for possibly filamentary emission features (Fig. 16) extending over 8" in the sky. At $z=2.4$, this corresponds to a projected size of 66 physical $\mathrm{kpc}$, i.e. the virial diameter of a halo with total mass $M \sim 4 \times 10^{11} M_{\odot}$. Without kinematical information, it is however difficult to determine if these extended features are associated with infall or outflows.

Other mechanisms can produce filamentary structures. In fact, in our simulations, the most common sign of filamentary CGM emission is associated with tidal features, e.g., dense gas stripped out of satellite galaxies, rather than the cold infall of gas. This could be the origin of the extended emission observed in our images. In particular the observed "bridge" between two of our bright extended sources, \#2411 and \#2433 might fall into this category (see also Rauch et al. 2011). Further theoretical and observational studies focusing on these sources, e.g., with the help of Integral Field Spectroscopy, might help reveal the detailed properties of the detected extended emission.

While much remains to be learnt about these systems, the key point is that our narrow-band survey has demonstrated the potential of finding and mapping the extended, cold circum-galactic medium around galaxies thanks to fluorescent boosting of the emission. This mechanism opens up a new window for the study of this important gas reservoir of high-redshift galaxies and to unraveling how galaxies receive their gas.

\section{UNCERTAINTIES AND LIMITATIONS}

\subsection{Foreground and AGN contamination}

What fraction of our $\operatorname{Ly} \alpha$ candidates could be foreground line emitting galaxies and how do this affects our results? Typically, the primary sources of contamination for highredshift NB Ly $\alpha$ surveys are foreground galaxies with strong [OII] 3726-3729 $\AA$ emission line doublet. For Ly $\alpha$ surveys at $2<z<3$, corresponding to $0.1<z<0.3$ for [OII], the typical fraction of foreground contamination is typically less than 5\% (e.g., Venemans et al. 2007), because of the much smaller volume and smaller EW associated with [OII] emitters. From our data, we cannot directly estimate the foreground contamination in our sample because of the lack of 
spectroscopic confirmation for the whole sample. The spectroscopic follow-up of about twenty among the brightest objects in our sample (Cantalupo et al., in prep.) clearly shows that they are Ly $\alpha$ emitters but the intrinsic faintness for the rest of our sample makes their direct confirmation more difficult.

Although foreground contamination could increase the number of detectable objects (affecting the luminosity function), it cannot produce our skewed EW distribution towards higher values. On the contrary, given their smaller EW, foreground objects would be expected to produce the opposite effect. Moreover, they would not show any correlation between their luminosity and the position of the quasar. Therefore, we believe that the presence of foregrounds in our sample does not affect our conclusions.

Unlike star-powered galaxies, active galactic nuclei (AGN) are able to produce EW well above the canonical $240 \AA$ value and might thus be "confused" with fluorescent emission. AGN can be identified by spectroscopic features (e.g., broad emission lines and the presence of the CIV doublet) or from X-ray coincident detections. Previous surveys have shown that AGN contamination is always very low, of the order of few percent (e.g., Guaita et al. 2010). It is in principle conceivable that there might be an increased number of AGN around our ultra-luminous quasar. Although clustering effects would increase the number of galaxies around the quasar, to our knowledge there are no indications in the literature for the increase, by a factor of at least five, in the AGN fraction in such environments, which would be necessary to explain our results in terms of AGN. This is corroborated by our preliminary spectroscopic confirmation for the bright part of our sample (Cantalupo et al., in prep.): only one object out of about twenty shows the presence of CIV emission. Therefore, although AGN might contribute to increasing the number of high EW objects in our sample, it is very unlikely that they could account for observed difference with the "blank-fields".

\subsection{Collisionally excited Ly $\alpha$}

Ly $\alpha$ produced by collisional excitation via energetic electrons, commonly called "Ly $\alpha$ cooling", is a mechanism that is in principle able to produce strong Ly $\alpha$ emission without associated star formation, i.e. similarly to quasar fluorescence. Unfortunately, collisionally excited Ly $\alpha$ emission has an exponential dependence on gas temperature (see e.g., Fig.1 in Cantalupo et al. 2008), which makes accurate predictions within current hydro-simulations very challenging (see Appendix B for discussion).

For the primary purpose of this study - finding protogalactic clouds and direct imaging of the cold CGM - it does not really matter if Ly $\alpha$ photons are produced by quasar fluorescence or by cooling due to gravitational collapse. From an observational point of view, Ly $\alpha$ cooling should be equally detectable away from bright quasars. If our sources are in fact powered by cooling instead of fluorescence, then the "blank-field" surveys at $2<z<3$ should already have detected such objects, and we should not see any particular difference between, e.g. our EW distribution and the one obtained by these surveys. On the other hand, cooling Ly $\alpha$ emission could explain why "blank-field" surveys do detect some objects with high EW away from bright quasars, including the large Ly $\alpha$ blobs. These sources would also then be present within our survey. However, given their low space density (e.g., Matsuda et al. 2004) we would expect that they are responsible for only a small fraction of our sample of high EW sources.

\subsection{Quasar effects}

The ionizing radiation from the quasar has the positive effect, for us, of increasing the ionization state, and thus the recombinant Ly $\alpha$ emission, of the gas. However, the same radiation could also have an important effect on the galaxyformation process itself around the quasar, e.g., reducing the gas cooling rate (see Cantalupo 2010) and thus effectively limiting gas accretion onto (proto-)galaxies and suppressing their SFRs. In principle, from the continuum properties of the sources in our survey it would be possible to search for a general star-forming deficit relative to the "blank-fields". However, the intrinsic faintness of our objects plus uncertain clustering effects would make this task very difficult in practice.

Nevertheless, let us assume for the moment that the galaxy-formation process would be "slowed-down" for a gas cloud while it is "illuminated" by the quasar, e.g. if the gas is completely ionized. In this case, the overall effect on the galaxies would depend on the quasar emission history compared to the galaxy-formation timescales. Within the current paradigm of quasar black-hole accretion the quasar luminous phase is expected to have a relatively short duration (e.g. a few tens of Myrs) followed by a much longer phase (e.g., a few Gyrs) of lower ultraviolet output (e.g., Di Matteo et al. 2008; see also Martini 2004 for a review). The dense gas, which should be associated with quasar fluorescent emission has a recombination time scale is very much shorter, of the order of $10^{5}-10^{6} \mathrm{yrs}$, than the quasar lifetime, and of cosmological timescales. This means that any previous bright phase of the quasar will be quickly "forgotten" once the quasar turns off, and proto-galactic clouds and galaxies should be able to recover their "normal" evolution before the current phase. At the same time, the star formation timescale probed by our deep $\mathrm{V}$ band image (centered on $\lambda \sim 1600 \AA$ ) is at least 200 Myrs (e.g., Leitherer et al. 1999), one order of magnitude larger than the expected quasar luminous phase. Therefore it is unlikely that the current bright phase is the cause of the apparent lack of star formation in our proto-galactic clouds candidates. Of course, we cannot exclude that our quasar had a very peculiar emission history. Future observational campaigns will be crucial to increase the the sample to a larger number of quasars and therefore reduce these uncertainties.

\section{SUMMARY AND CONCLUSIONS}

We have presented a deep survey for fluorescent Ly $\alpha$ emission in a large cosmological volume $\left(\sim 5 \times 10^{3}\right.$ comoving Mpc) centered on the hyperluminous $z=2.4$ quasar HE0109-3518. With the help of a custom-made, narrowband filter mounted on the VLT/FORS spectrograph, we were able to photometrically select 98 Ly $\alpha$ candidates down to a detection limit of about $4 \times 10^{-18} \mathrm{erg} \mathrm{s}^{-1} \mathrm{~cm}^{-2}$ (corresponding to $L_{L y \alpha} \sim 2 \times 10^{41}$ erg $s^{-1}$ at $z=2.4$ ). 
The properties of these $\operatorname{Ly} \alpha$ sources in terms of their: i) equivalent width distribution, ii) shape of the luminosity function and, iii) the variation of their luminosity with projected distance from the quasar, all indicate that our sample is consistent with having much of the Ly $\alpha$ emission originating in fluorescent reprocessing of quasar radiation.

In particular, the main evidence supporting the fluorescent origin of our sample are:

- The large number of objects with rest-frame $\mathrm{EW}_{0}>$ $240 \AA$ (18 sources out of 98$)$, commonly assumed to be the limit for Ly $\alpha$ emission powered by Population II stars (e.g., Schaerer 2002). Independent of luminosity, the fraction of such objects appears to be about $20 \%$, which is about a factor of four larger than "blank-field" surveys (e.g., Ciardullo et al. 2012, Grove et al. 2009) or surveys around radiogalaxies (Venemans et al. 2007) at similar redshifts. Moreover, the whole shape of our equivalent width distribution is skewed toward higher values, with an exponential scale that is about double that found in the "blank-fields".

- The steeper faint-end of our LF with respect to the "blank-fields" surveys with a corresponding increase in the source number density by a factor of about five compared to these studies. The steepening of the LF is well reproduced by a radiative transfer simulation of fluorescent emission around quasars. The simulation suggests that the difference with the "blank-field" surveys may be fully explained by fluorescent boosting of gas-rich but intrinsically faint Ly $\alpha$ emitters.

- The increase in the average luminosity of the sources at smaller (projected) distances from the quasar. Although this effect could also be caused by clustering of bright sources around the quasar, our simulations suggest that the observed trend is very similar to the theoretical expectations for fluorescent emission.

Fluorescent emission gives us a unique opportunity to directly image, in emission, dense, high-redshift gas independent of any associated star formation. In particular, we identify three categories of objects associated with fluorescent emission in our study:

- Proto-galactic or "dark" clouds, i.e. gas-rich objects with little or no associated star-formation. Candidates for these sources are represented by the 12 objects without detectable continuum and with lower limits on their EW higher above $240 \AA$. They are typically compact or unresolved with $L \lesssim 10^{42} \mathrm{erg} \mathrm{s}^{-1}$. A V-band image stack of these objects did not show any continuum detection above $1 \sigma(V \sim 30.3)$, implying a combined constraint on their $\mathrm{EW}$ of $\mathrm{EW}_{0}>800 \AA$ $(1 \sigma)$. This effectively rules out Ly $\alpha$ powered by internal star formation. A comparison with our radiative transfer simulations suggests that these sources would have maximal HI column densities around or slightly below the DLA threshold $\left(2 \times 10^{20} \mathrm{~cm}^{-2}\right)$ if they were observed away from the quasar. These may therefore be the analogues of absorption systems which have also been suggested to probe such a "proto-galactic phase" (e.g., Fumagalli et al. 2011, Cooke et al. 2011). Observing these systems in emission - rather than in absorption - allows us to readily discriminate between truly isolated clouds and sub-components of larger, galactic or circum-galactic gas reservoirs. Our narrow-band imaging provides indications for the existence of compact and isolated proto-galactic or "dark" clouds opening up a new observational window for the study of the early phases of galaxy formation. Assuming that these objects are almost fully ionized by the intense quasar radiation, we can directly derive an estimate or their total gas mass, namely $M_{\text {gas }} \sim 10^{9} M_{\odot}$. From the non-detection in the V-band stack, we can furthermore estimate an upper limit on their average star formation rate of $\mathrm{SFR}<10^{-2} M_{\odot} \mathrm{yr}^{-1}$. This implies that their star formation efficiency is lower than $\mathrm{SFE}<10^{-11} \mathrm{yr}^{-1}$ (or, equivalently, their gas consumption time is larger than 100 Gyr), several times below the SFE of gas-rich dwarf galaxies in the local Universe (e.g., Geha et al. 2006) and two hundred times lower than in typical massive star-forming galaxies at these redshifts (Daddi et al, 2010, Genzel et al 2010). These objects are therefore the best candidates for proto-galactic clouds or "dark" galaxies at high-redshift suggested by recent theoretical studies (e.g., Bouché et al. 2010, Gnedin \& Kravtsov 2010, Krumholz \& Dekel 2011, Kuhlen et al. 2012).

- Intrinsically faint Lya emitting galaxies fluorescently boosted above our detection threshold. As suggested by our radiative transfer simulations, these sources are the main component producing the observed e-folding scale increase in the EW distribution and the steepening of the LF. In particular, their implied SFRs are about one order of magnitude smaller than the values we could have detected in absence of quasar fluorescence.

- Extended, cold circum-galactic medium emission. At least three of the six bright sources with $\mathrm{EW}_{0}>240 \AA$ and continuum detection in our sample show extended, possibly filamentary fluorescent emission. In particular, one object (\#1159) presents signs of several filaments connected to a bright central source. The size and morphology of these structures are compatible with the expectations from theoretical and numerical models for cold streams feeding highredshift galaxies (e.g., Dekel et al. 2009). Our current data and the lack of kinematical information cannot however rule out a different origin for this cold and dense circum-galactic gas, e.g., tidal stripping from gas-rich satellite galaxies (as commonly seen in our simulations).

After several years of attempts to detect fluorescent Ly $\alpha$ emission, our results demonstrate the potential of a carefully designed (very-)narrow-band survey around bright quasars to discover and study this new category of sources. Several important factors have contributed to this success. As we have shown, a large survey volume, comparably deep narrow-band and continuum imaging, as well as sophisticated numerical models including radiative transfer effects, are fundamental to detect, recognize and interpret fluorescent emission. Still, the success of such a fluorescence survey might depend on the emitting history and properties of the source that provides the "illumination", i.e. the quasar. In this respect, our survey suggests that for HE0109-3518 at least, the proximity effect extends on much larger angular scale than just our line-of-sight, the intrinsic limit of absorption-line studies. Object by object variations, including clustering and cosmic variance, might however play an important role and observational campaigns targeting a larger number of quasars will thus be important in the future.

By stimulating fluorescent emission in neutral gas, the 
quasars are literally shedding light on the hitherto obscure early stages of galaxy-formation and on the way in which galaxies acquire their gas. With the present study, we make a crucial step towards revealing and understanding how to take full advantage of this unique opportunity.

\section{ACKNOWLEDGMENTS}

We are grateful to the staff of Paranal, Chile for their excellent support. We thank the referee for a very detailed and careful review of the manuscript and for constructive questions and comments. SC thanks J. Xavier Prochaska for carefully reading an earlier version of the manuscript and providing suggestions that improved the presentation of this work and George Becker, Mattew Hayes, Piero Madau, Richard McMahon, Michael Rauch, Debora Sijacki, Dan Stark and Gabor Worsek for many very useful discussions. The hydrodynamical simulations used in this work were performed using the Darwin Supercomputer of the University of Cambridge High Performance Computing Service (http://www.hpc.cam.ac.uk/), provided by Dell Inc. using Strategic Research Infrastructure Funding from the Higher Education Funding Council for England. SC acknowledges support from NSF grants AST-1010004. This research was supported in part by the National Science Foundation under Grant No. PHY11-25915.

\section{REFERENCES}

Barnes, L. A., Haehnelt, M. G., Tescari, E., \& Viel, M. 2011, MNRAS, 416, 1723

Bertin, E., \& Arnouts, S. 1996, A\&AS, 117, 393

Bolton, J. S., Haehnelt, M. G., Viel, M., \& Springel, V. 2005, MNRAS, 357, 1178

Bouché, N., et al. 2010, ApJ, 718, 1001

Cantalupo, S., Porciani, C., Lilly, S. J., \& Miniati, F. 2005, ApJ, 628, 61

Cantalupo, S., Lilly, S. J., \& Porciani, C. 2007, ApJ, 657, 135

Cantalupo, S., Porciani, C., \& Lilly, S. J. 2008, ApJ, 672, 48

Cantalupo, S. 2010, MNRAS, 403, L16

Cantalupo, S., \& Porciani, C. 2011, MNRAS, 411, 1678

Carswell, R. F., Becker, G. D., Jorgenson, R. A., Murphy,

M. T., \& Wolfe, A. M. 2012, MNRAS, 2703

Charlot, S., \& Fall, S. M. 1993, ApJ, 415, 580

Ciardullo, R., et al. 2012, ApJ, 744, 110

Cooke, R., Pettini, M., Steidel, C. C., Rudie, G. C., \& Jorgenson, R. A. 2011, MNRAS, 412, 1047

Cooke, R., Pettini, M., \& Murphy, M. 2012, arXiv:1201.1004

Crampton, D., \& Lilly, S.J. 1999, In "Photometric Redshifts and the Detection of High Redshift Galaxies", ASP Conference Series, Vol. 191, ed. Ray Weymann, Lisa Storrie-Lombardi, Marcin Sawicki, and Robert Brunner, p.229

Croom, S. M., \& Shanks, T. 1999, MNRAS, 303, 411

Daddi, E., et al. 2010, ApJ, 713, 686

Dall'Aglio, A., Wisotzki, L., \& Worseck, G. 2008, A\&A, 491, 465
Dawson, S., et al. 2004, ApJ, 617, 707

Dekel, A., \& Birnboim, Y. 2006, MNRAS, 368, 2

Dekel, A., Birnboim, Y., Engel, G., et al. 2009, Nature, 457, 451

Dijkstra, M., Haiman, Z., \& Spaans, M. 2006, ApJ, 649, 14

Dijkstra, M., \& Loeb, A. 2009, MNRAS, 400, 1109

D'Odorico, V., et al. 2008, MNRAS, 389, 1727

Dubois, Y., \& Teyssier, R. 2008, A\&A, 477, 79

Faucher-Giguère, C.-A., Kereš, D., Dijkstra, M., Hernquist, L., \& Zaldarriaga, M. 2010, ApJ, 725, 633

Falder, J. T., et al. 2011, ApJ, 735, 123

Fardal, M. A., et al. 2001, ApJ, 562, 605

Feldman, G. J., \& Cousins, R. D. 1998, PhRvDx, 57, 3873

Ferland, G. J., et al. 1998, PASP, 110, 761

Fynbo, J. P. U., Ledoux, C., Möller, P., Thomsen, B., \& Burud, I. 2003, A\&A, 407, 147

Francis, P. J., \& Bland-Hawthorn, J. 2004, MNRAS, 353, 301

Francis, P. J., \& McDonnell, S. 2006, MNRAS, 370, 1372

Fumagalli, M., O’Meara, J. M., \& Prochaska, J. X. 2011, Science, 334, 1245

Fumagalli, M., et al. 2011, MNRAS, 418, 1796

Furlanetto, S. R., Schaye, J., Springel, V., \& Hernquist, L. 2005, ApJ, 622, 7

Gavazzi, G., et al. 2008, A\&A, 482, 43

Geha, M., Blanton, M. R., Masjedi, M., \& West, A. A. 2006, ApJ, 653, 240

Gehrels, N. 1986, ApJ, 303, 336

Genzel, R., et al. 2010, MNRAS, 407, 2091

Giovanelli, R., et al. 2005, AJ, 130, 2598

Gnedin, N. Y., \& Kravtsov, A. V. 2010, ApJ, 714, 287

Gnedin, N. Y., \& Kravtsov, A. V. 2011, ApJ, 728, 88

Goerdt, T., Dekel, A., Sternberg, A., et al. 2010, MNRAS, 407, 613

Gonçalves, T. S., Steidel, C. C., \& Pettini, M. 2008, ApJ, 676,816

Gould, A., \& Weinberg, D. H. 1996, ApJ, 468, 462

Guaita, L., et al. 2010, ApJ, 714, 255

Guaita, L., et al. 2011, ApJ, 733, 114

Grove, L. F., et al. 2009, A\&A, 497, 689

Haardt, F., \& Madau, P. 2012, ApJ, 746, 125

Haiman, Z., Spaans, M., \& Quataert, E., 2000, ApJL, 537, L5

Haiman, Z., \& Rees, M. J. 2001, ApJ, 556, 87

Hayes, M., et al. 2010, Nature, 464, 562

Heckman, T. M., Lehnert, M. D., Miley, G. K., \& van Breugel, W. 1991, ApJ, 381, 373

Hennawi, J. F., et al. 2006, ApJ, 651, 61

Hennawi, J. F., \& Prochaska, J. X. 2007, ApJ, 655, 735

Hennawi, J. F., Prochaska, J. X., Kollmeier, J., \& Zheng, Z. 2009, ApJL, 693, L49

Hogan, C. J., \& Weymann, R. J. 1987, MNRAS, 225, 1P

Jakobsen, P., Jansen, R. A., Wagner, S., \& Reimers, D. 2003, A\&A, 397, 891

Kirkman, D., \& Tytler, D. 2008, MNRAS, 391, 1457

Kennicutt, R. C., Jr. 1998, ApJ, 498, 541

Kereš, D., Katz, N., Fardal, M., Davé, R., \& Weinberg, D. H. 2009, MNRAS, 395, 160

Kollmeier, J. A., Zheng, Z., Davé, R., et al. 2010, ApJ, 708, 1048

Komatsu, E., et al. 2011, ApJS, 192, 18

Krumholz, M. R., \& Dekel, A. 2011, arXiv:1106.0301 
Krumholz, M. R., McKee, C. F., \& Tumlinson, J. 2009, ApJ, 699, 850

Kuhlen, M., Krumholz, M. R., Madau, P., Smith, B. D., \& Wise, J. 2012, ApJ, 749, 36

Leitherer, C., et al. 1999, ApJS, 123, 3

Malhotra, S., \& Rhoads, J. E. 2002, ApJL, 565, L71

Martini, P. 2004, Coevolution of Black Holes and Galaxies, 169

Marziani, P., Sulentic, J. W., Stirpe, G. M., Zamfir, S., \&

Calvani, M. 2009, A\&A, 495, 83

Matsuda, Y., et al. 2004, AJ, 128, 569

Meurer, G. R., Heckman, T. M., \& Calzetti, D. 1999, ApJ, 521,64

Mo, H. J., Mao, S., \& White, S. D. M. 1998, MNRAS, 295, 319

Nilsson, K. K., Fynbo, J. P. U., Møller, P., Sommer-Larsen, J., \& Ledoux, C. 2006, A\&A, 452, L23

Nilsson, K. K., Tapken, C., Møller, P., et al. 2009, A\&A, 498, 13

Ocvirk, P., Pichon, C., \& Teyssier, R. 2008, MNRAS, 390, 1326

Osterbrock, D. E. 1989, Research supported by the University of California, John Simon Guggenheim Memorial Foundation, University of Minnesota, et al. Mill Valley, CA, University Science Books, 1989, 422 p.,

Otí-Floranes, H., \& Mas-Hesse, J. M. 2010, A\&A, 511, A61

Ouchi, M., et al. 2008, ApJS, 176, 301

Penprase, B. E., Prochaska, J. X., Sargent, W. L. W., ToroMartinez, I., \& Beeler, D. J. 2010, ApJ, 721, 1

Prescott, M. K. M., Dey, A., \& Jannuzi, B. T. 2012, ApJ, 748, 125

Rafelski, M., Wolfe, A. M., \& Chen, H.-W. 2011, ApJ, 736, 48

Raiter, A., Schaerer, D., \& Fosbury, R. A. E. 2010, A\&A, 523, A64

Rauch, M., Miralda-Escude, J., Sargent, W. L. W., et al. 1997, ApJ, 489, 7

Rauch, M. 1998, ARA\&A, 36, 267

Rauch, M., et al. 2008, ApJ, 681, 856

Rauch, M., et al. 2011, MNRAS, 418, 1115

Rees, M. J., \& Ostriker, J. P. 1977, MNRAS, 179, 541

Rosdahl, J., \& Blaizot, J. 2011, arXiv:1112.4408

Saito, T., Shimasaku, K., Okamura, S., et al. 2006, ApJ, 648, 54

Scarlata, C., et al. 2009, ApJ, 706, 1241

Schaerer, D. 2002, A\&A, 382, 28

Schlegel, D. J., Finkbeiner, D. P., \& Davis, M. 1998, ApJ, 500,525

Shemmer, O., et al. 2004, ApJ, 614, 547

Schaye, J. 2001, ApJ, 559, 507

Schiminovich, D., et al. 2010, MNRAS, 408, 919

Scott, J., Bechtold, J., Morita, M., Dobrzycki, A., \& Kulkarni, V. P. 2002, ApJ, 571, 665

Serber, W., Bahcall, N., Ménard, B., \& Richards, G. 2006, ApJ, 643, 68

Shimasaku, K., et al. 2006, PASJ, 58, 313

Steidel, C. C., et al. 2011, ApJ, 736, 160

Steidel, C. C., et al. 2000, ApJ, 532, 170

Telfer, R. C., Zheng, W., Kriss, G. A., \& Davidsen, A. F. 2002, ApJ, 565, 773

Teyssier, R. 2002, A\&A, 385, 337

Trainor, R. F., \& Steidel, C. C. 2012, arXiv:1204.3636 van de Voort, F., \& Schaye, J. 2012, MNRAS, 2882

Venemans, B. P., et al. 2007, A\&A, 461, 823

White, S. D. M., \& Rees, M. J. 1978, MNRAS, 183, 341

Wolfe, A. M., Gawiser, E., \& Prochaska, J. X. 2005, ARA\&A, 43, 861x

Worseck, G., Fechner, C., Wisotzki, L., \& Dall'Aglio, A. 2007, A\&A, 473, 805

Yang, Y., et al. 2006, ApJ, 640, 539

Yang, Y., Zabludoff, A., Tremonti, C., Eisenstein, D., \& Davé, R. 2009, ApJ, 693, 1579

Zheng, Z., Cen, R., Weinberg, D., Trac, H., \& MiraldaEscudé, J. 2011, ApJ, 739, 62 
APPENDIX A: LIST OF CONTINUUM DETECTED OBJECTS WITH EW W $_{0} 240 \AA$

Table A1: Position and properties Ly $\alpha$ candidates with continuum detection and $\mathrm{EW}_{0}<240 \AA$ (see Table 2)

\begin{tabular}{|c|c|c|c|c|c|c|c|c|}
\hline Id & $\begin{array}{c}\mathrm{RA} \\
(\mathrm{J} 2000)\end{array}$ & $\begin{array}{c}\text { DEC } \\
(\mathrm{J} 2000)\end{array}$ & $\begin{array}{l}\mathrm{NB} \\
(\mathrm{AB})\end{array}$ & $\begin{array}{c}\mathrm{V} \\
(\mathrm{AB})\end{array}$ & $\begin{array}{c}\mathrm{B} \\
(\mathrm{AB})\end{array}$ & $\begin{array}{c}\mathrm{F} \\
10^{-17} \mathrm{erg} \mathrm{s}^{-1} \mathrm{~cm}^{-2}\end{array}$ & $\begin{array}{c}\mathrm{L}_{\text {Ly }} \\
10^{42} \mathrm{erg} \mathrm{s}^{-1}\end{array}$ & $\begin{array}{c}\mathrm{EW}_{0} \\
\AA\end{array}$ \\
\hline 2433 & $1: 11: 33.85$ & $-35: 01: 12.4$ & $23.32_{-0.08}^{+0.09}$ & $25.35_{-0.11}^{+0.12}$ & $26.3_{-0.1}^{+0.11}$ & $12.22 \pm 0.97$ & $5.68 \pm 0.45$ & $218 \pm 27$ \\
\hline 248 & $1: 11: 58.01$ & $-35: 06: 08.9$ & $24.85_{-0.08}^{+0.08}$ & $28.05_{-0.23}^{+0.28}$ & $>28.49$ & $2.99 \pm 0.21$ & $1.39 \pm 0.10$ & $207 \pm 45$ \\
\hline 973 & $1: 11: 40.02$ & $-35: 04: 30.7$ & $24.77_{-0.08}^{+0.08}$ & $27.88_{-0.24}^{+0.30}$ & $>28.58$ & $3.21 \pm 0.23$ & $1.49 \pm 0.11$ & $192 \pm 45$ \\
\hline 558 & $1: 11: 28.50$ & $-35: 05: 22.7$ & $25.44_{-0.11}^{+0.08}$ & $28.42_{-0.21}^{+0.24}$ & $>28.89$ & $1.73 \pm 0.18$ & $0.81 \pm 0.08$ & $168 \pm 35$ \\
\hline 2232 & $1: 11: 38.52$ & $-35: 00: 39.4$ & $25.75_{-0.11}^{+0.11}$ & $28.65_{-0.25}^{+0.32}$ & $28.31_{-0.25}^{+0.31}$ & $1.30 \pm 0.14$ & $0.61 \pm 0.06$ & $155 \pm 40$ \\
\hline 2360 & $1: 11: 42.06$ & $-35: 00: 58.7$ & $24.43_{-0.06}^{+0.06}$ & $27.29_{-0.12}^{+0.13}$ & $27.88_{-0.25}^{+0.30}$ & $4.40 \pm 0.24$ & $2.04 \pm 0.11$ & $149 \pm 15$ \\
\hline 1946 & $1: 11: 38.58$ & $-35: 00: 07.4$ & $24.19_{-0.10}^{+0.06}$ & $25.38_{-0.12}^{+0.13}$ & $26.68_{-0.13}^{+0.14}$ & $5.48 \pm 0.48$ & $2.55 \pm 0.22$ & $147 \pm 24$ \\
\hline 1198 & 1:11:31.04 & $-35: 03: 55.3$ & $23.85_{-0.05}^{+0.05}$ & $26.36_{-0.09}^{+0.09}$ & $26.60_{-0.15}^{+0.16}$ & $7.50 \pm 0.34$ & $3.48 \pm 0.16$ & $145 \pm 20$ \\
\hline 1899 & $1: 11: 36.67$ & $-35: 02: 59.1$ & $23.63_{-0.09}^{+0.09}$ & $25.39_{-0.09}^{+0.09}$ & $26.22_{-0.11}^{+0.10}$ & $9.18 \pm 0.73$ & $4.27 \pm 0.34$ & $144 \pm 18$ \\
\hline 2818 & $1: 11: 51.42$ & $-35: 02: 05.8$ & $23.38_{-0.07}^{+0.08}$ & $25.25_{-0.06}^{+0.07}$ & $25.96_{-0.09}^{+0.09}$ & $11.56 \pm 0.82$ & $5.37 \pm 0.38$ & $139 \pm 12$ \\
\hline 2965 & $1: 11: 31.74$ & $-35: 02: 20.5$ & $25.54_{-0.09}^{+0.010}$ & $28.17_{-0.25}^{+0.32}$ & $>28.46$ & $1.58 \pm 0.14$ & $0.73 \pm 0.06$ & $118 \pm 30$ \\
\hline 2958 & 1:11:40.94 & $-35: 02: 22.5$ & $24.66_{-0.07}^{+0.07}$ & $26.52_{-0.09}^{+0.09}$ & $27.1_{-0.20}^{+0.23}$ & $3.56 \pm 0.22$ & $1.65 \pm 0.10$ & $117 \pm 23$ \\
\hline 2345 & $1: 11: 33.62$ & $-35: 01: 03.0$ & $24.58_{-0.12}^{+0.13}$ & $25.81_{-0.1}^{+0.05}$ & $26.86_{-0.15}^{+0.16}$ & $3.83 \pm 0.43$ & $1.78 \pm 0.20$ & $112 \pm 19$ \\
\hline 1172 & $1: 11: 51.25$ & $-35: 03: 59.6$ & $24.39_{-0.12}^{+0.13}$ & $25.63_{-0.13}^{+0.14}$ & $26.66_{-0.14}^{+0.15}$ & $4.56 \pm 0.51$ & $2.12 \pm 0.24$ & $111 \pm 20$ \\
\hline 510 & 1:11:35.90 & $-35: 05: 28.9$ & $25.89_{-0.14}^{+0.16}$ & $28.44_{-0.22}^{+0.26}$ & $28.12_{-0.24}^{+0.30}$ & $1.15 \pm 0.16$ & $0.53 \pm 0.07$ & $110 \pm 25$ \\
\hline 1487 & $1: 11: 36.40$ & $-35: 03: 13.5$ & $24.70_{-0.14}^{+0.14}$ & $26.11_{-0.13}^{+0.14}$ & $26.99_{-0.20}^{+0.23}$ & $3.43 \pm 0.44$ & $1.59 \pm 0.21$ & $109 \pm 25$ \\
\hline 2547 & $1: 11: 37.98$ & $-35: 01: 28.6$ & $24.29_{-0.05}^{+0.05}$ & $26.94_{-0.12}^{+0.13}$ & $26.83_{-0.15}^{+0.16}$ & $5.00 \pm 0.23$ & $2.32 \pm 0.10$ & $107 \pm 17$ \\
\hline 2251 & $1: 11: 29.99$ & $-35: 00: 44.7$ & $24.95_{-0.14}^{+0.05}$ & $26.38_{-0.13}^{+0.14}$ & $27.2_{-0.19}^{+0.22}$ & $2.72 \pm 0.35$ & $1.26 \pm 0.16$ & $102 \pm 23$ \\
\hline 2804 & $1: 11: 51.42$ & $-35: 02: 03.2$ & $24.44_{-0.12}^{+0.13}$ & $26.18_{-0.12}^{+0.13}$ & $26.7_{-0.14}^{+0.16}$ & $4.35 \pm 0.49$ & $2.02 \pm 0.23$ & $95 \pm 17$ \\
\hline 1881 & $1: 11: 46.41$ & $-34: 59: 56.5$ & $25.52_{-0.09}^{+0.09}$ & $27.92_{-0.24}^{+0.13}$ & $28.16_{-0.32}^{+0.43}$ & $1.61 \pm 0.13$ & $0.75 \pm 0.06$ & $94 \pm 22$ \\
\hline 2629 & $1: 11: 41.16$ & $-35: 01: 38.1$ & $24.06_{-0.09}^{+0.10}$ & $25.73_{-0.08}^{+0.09}$ & $26.28_{-0.10}^{+0.11}$ & $6.18 \pm 0.54$ & $2.87 \pm 0.25$ & $92 \pm 10$ \\
\hline 2594 & $1: 11: 35.80$ & $-35: 01: 29.3$ & $24.68_{-0.14}^{+0.15}$ & $27.04_{-0.18}^{+0.21}$ & $>27.99$ & $3.49 \pm 0.45$ & $1.62 \pm 0.21$ & $90 \pm 18$ \\
\hline 2496 & $1: 11: 37.72$ & $-35: 01: 17.0$ & $25.21_{-0.10}^{+0.14}$ & $27.27_{-0.15}^{+0.18}$ & $27.33_{-0.21}^{+0.25}$ & $2.14 \pm 0.19$ & $1.00 \pm 0.09$ & $73 \pm 18$ \\
\hline 567 & $1: 11: 48.19$ & $-35: 05: 22.0$ & $25.77_{-0.11}^{+0.10}$ & $27.92_{-0.14}^{+0.15}$ & $>29.14$ & $1.28 \pm 0.13$ & $0.59 \pm 0.06$ & $72 \pm 10$ \\
\hline 1837 & $1: 11: 39.74$ & $-34: 59: 52.7$ & $24.96_{-0.08}^{+0.08}$ & $26.92_{-0.10}^{+0.14}$ & $27.05_{-0.15}^{+0.16}$ & $2.70 \pm 0.19$ & $1.25 \pm 0.09$ & $71 \pm 11$ \\
\hline 2682 & $1: 11: 42.31$ & $-35: 01: 46.1$ & $25.18_{-0.10}^{+0.08}$ & $26.71_{-0.10}^{+0.10}$ & $27.17_{-0.20}^{+0.24}$ & $2.20 \pm 0.19$ & $1.02 \pm 0.09$ & $70 \pm 15$ \\
\hline 525 & $1: 11: 58.63$ & $-35: 05: 29.8$ & $25.80_{-0.12}^{+0.12}$ & $27.89_{-0.2}^{+0.24}$ & $>28.64$ & $1.24 \pm 0.13$ & $0.58 \pm 0.06$ & $68 \pm 14$ \\
\hline 1649 & $1: 11: 41.78$ & $-35: 03: 34.9$ & $25.90_{-0.13}^{+0.14}$ & $27.99_{-0.27}^{+0.35}$ & $>28.29$ & $1.13 \pm 0.14$ & $0.53 \pm 0.06$ & $68 \pm 19$ \\
\hline 1413 & $1: 11: 45.24$ & $-35: 03: 06.6$ & $25.26_{-0.08}^{+0.08}$ & $27.35_{-0.16}^{+0.18}$ & $>28.56$ & $2.05 \pm 0.15$ & $0.95 \pm 0.07$ & $67 \pm 10$ \\
\hline 1931 & $1: 11: 28.37$ & $-35: 00: 12.4$ & $26.51_{-0.17}^{+0.08}$ & $28.59_{-0.33}^{+0.10}$ & $>28.71$ & $0.65 \pm 0.10$ & $0.30 \pm 0.05$ & $67 \pm 24$ \\
\hline 2076 & $1: 11: 32.03$ & $-35: 00: 25.9$ & $26.81_{-0.16}^{+0.17}$ & $28.88_{-0.28}^{+0.36}$ & $>29.19$ & $0.49 \pm 0.07$ & $0.23 \pm 0.03$ & $66 \pm 20$ \\
\hline 1538 & $1: 11: 46.44$ & $-35: 03: 22.4$ & $25.14_{-0.18}^{+0.10}$ & $27.15_{-0.27}^{+0.35}$ & $>28.31$ & $2.29 \pm 0.38$ & $1.06 \pm 0.18$ & $62 \pm 19$ \\
\hline 963 & $1: 11: 29.86$ & $-35: 04: 35.7$ & $25.24_{-0.10}^{+0.18}$ & $26.53_{-0.09}^{+0.09}$ & $27.06_{-0.18}^{+0.20}$ & $2.08 \pm 0.18$ & $0.97 \pm 0.09$ & $60 \pm 11$ \\
\hline 791 & $1: 11: 57.22$ & $-35: 04: 53.0$ & $26.36_{-0.14}^{+0.16}$ & $28.34_{-0.19}^{+0.23}$ & $28.18_{-0.24}^{+0.29}$ & $0.74 \pm 0.10$ & $0.35 \pm 0.05$ & $60 \pm 13$ \\
\hline 380 & $1: 11: 41.15$ & $-35: 05: 51.0$ & $26.12_{-0.15}^{+0.14}$ & $28.1_{-0.23}^{+0.29}$ & $>28.55$ & $0.93 \pm 0.13$ & $0.43 \pm 0.06$ & $60 \pm 15$ \\
\hline 471 & $1: 11: 53.11$ & $-35: 05: 35.9$ & $25.72_{-0.11}^{+0.10}$ & $27.69_{-0.16}^{+0.18}$ & $>28.71$ & $1.34 \pm 0.14$ & $0.62 \pm 0.07$ & $59 \pm 10$ \\
\hline 1537 & $1: 11: 46.38$ & $-35: 03: 21.6$ & $25.21_{-0.17}^{+0.11}$ & $27.15_{-0.27}^{+0.10}$ & $>28.31$ & $2.14 \pm 0.34$ & $1.00 \pm 0.16$ & $57 \pm 17$ \\
\hline 683 & $1: 11: 44.21$ & $-35: 04: 59.2$ & $23.76_{-0.08}^{+0.08}$ & $24.71_{-0.05}^{+0.05}$ & $25.45_{-0.11}^{+0.12}$ & $8.15 \pm 0.58$ & $3.78 \pm 0.27$ & $55 \pm 6$ \\
\hline 2004 & $1: 11: 37.64$ & $-35: 00: 15.4$ & $25.56_{-0.10}^{+0.08}$ & $27.43_{-0.17}^{+0.05}$ & $>28.38$ & $1.55 \pm 0.15$ & $0.72 \pm 0.07$ & $53 \pm 9$ \\
\hline 1574 & $1: 11: 29.77$ & $-35: 03: 24.8$ & $25.21_{-0.10}^{+0.10}$ & $27.07_{-0.16}^{+0.19}$ & $>28.05$ & $2.14 \pm 0.21$ & $1.00 \pm 0.10$ & $52 \pm 9$ \\
\hline 2714 & 1:11:41.74 & $-35: 01: 49.3$ & $26.16_{-0.18}^{+0.20}$ & $27.96_{-0.20}^{+0.24}$ & $>28.59$ & $0.89 \pm 0.15$ & $0.41 \pm 0.07$ & $49 \pm 11$ \\
\hline 327 & $1: 11: 46.00$ & $-35: 05: 55.9$ & $24.25_{-0.09}^{0.10}$ & $24.93_{-0.06}^{+0.06}$ & $25.80_{-0.13}^{+0.14}$ & $5.19 \pm 0.46$ & $2.41 \pm 0.21$ & $49 \pm 6$ \\
\hline 246 & $1: 11: 46.93$ & $-35: 06: 08.2$ & $25.19_{-0.13}^{+0.14}$ & $26.98_{-0.21}^{+0.00}$ & $27.58_{-0.29}^{+0.38}$ & $2.18 \pm 0.26$ & $1.01 \pm 0.12$ & $48 \pm 10$ \\
\hline 1381 & $1: 11: 45.11$ & $-34: 59: 31.4$ & $24.78_{-0.11}^{+0.13}$ & $25.89_{-0.11}^{+0.13}$ & $26.41_{-0.14}^{+0.15}$ & $3.18 \pm 0.33$ & $1.48 \pm 0.15$ & $48 \pm 8$ \\
\hline 2105 & $1: 11: 40.48$ & $-35: 00: 30.4$ & $24.76_{-0.12}^{0.13}$ & $25.70_{-0.10}^{+0.11}$ & $26.33_{-0.19}^{+0.22}$ & $3.24 \pm 0.37$ & $1.51 \pm 0.17$ & $46 \pm 10$ \\
\hline 2187 & $1: 11: 59.42$ & $-35: 00: 39.3$ & $25.40_{-0.08}^{+0.08}$ & $27.11_{-0.14}^{+0.15}$ & $>28.31$ & $1.80 \pm 0.13$ & $0.84 \pm 0.06$ & $44 \pm 6$ \\
\hline 2231 & $1: 11: 59.30$ & $-35: 00: 38.8$ & $25.20_{-0.07}^{+0.07}$ & $26.91_{-0.11}^{+0.14}$ & $>28.38$ & $2.16 \pm 0.14$ & $1.00 \pm 0.06$ & $44 \pm 4$ \\
\hline 1540 & $1: 11: 46.52$ & $-35: 03: 18.5$ & $24.25_{-0.12}^{0.13}$ & $25.23_{-0.08}^{+0.08}$ & $25.77_{-0.17}^{+0.20}$ & $5.19 \pm 0.59$ & $2.41 \pm 0.27$ & $43 \pm 8$ \\
\hline 2089 & $1: 11: 42.47$ & $-35: 00: 25.4$ & $25.94_{-0.10}^{+0.11}$ & $27.36_{-0.12}^{+0.08}$ & $27.51_{-0.19}^{+0.22}$ & $1.09 \pm 0.11$ & $0.51 \pm 0.05$ & $40 \pm 8$ \\
\hline 1514 & $1: 11: 52.17$ & $-35: 03: 15.6$ & $24.48_{-0.12}^{0.12}$ & $24.85_{-0.06}^{+0.06}$ & $25.81_{-0.13}^{+0.19}$ & $4.20 \pm 0.44$ & $1.95 \pm 0.20$ & $38 \pm 5$ \\
\hline 1982 & $1: 11: 37.44$ & $-35: 00: 13.1$ & $25.83_{-0.14}^{+0.15}$ & $27.4_{-0.17}^{+0.20}$ & $>28.39$ & $1.21 \pm 0.16$ & $0.56 \pm 0.07$ & $37 \pm 7$ \\
\hline
\end{tabular}


Table A1 (continued)

\begin{tabular}{ccccccccc}
\hline \hline Id & $\begin{array}{c}\text { RA } \\
(\mathrm{J} 2000)\end{array}$ & $\begin{array}{c}\mathrm{DEC} \\
(\mathrm{J} 2000)\end{array}$ & $\begin{array}{c}\mathrm{NB} \\
(\mathrm{AB})\end{array}$ & $\begin{array}{c}\mathrm{V} \\
(\mathrm{AB})\end{array}$ & $\begin{array}{c}\mathrm{B} \\
(\mathrm{AB})\end{array}$ & $\begin{array}{c}\mathrm{F}_{L y \alpha} \\
10^{-17} \mathrm{erg} \mathrm{s}^{-1} \mathrm{~cm}^{-2}\end{array}$ & $\begin{array}{c}\mathrm{L}_{L y \alpha} \\
10^{42} \mathrm{erg} \mathrm{s}^{-1}\end{array}$ & $\begin{array}{c}\mathrm{EW}_{0} \\
\AA\end{array}$ \\
\hline 2633 & $1: 11: 32.45$ & $-35: 01: 34.1$ & $25.90_{-0.12}^{+0.13}$ & $27.38_{-0.13}^{+0.15}$ & $27.7_{-0.23}^{+0.28}$ & $1.13 \pm 0.13$ & $0.53 \pm 0.06$ & $33 \pm 5$ \\
2342 & $1: 11: 43.93$ & $-35: 00: 56.3$ & $26.24_{-0.17}^{+0.19}$ & $27.7_{-0.21}^{+0.25}$ & $>28.39$ & $0.83 \pm 0.13$ & $0.39 \pm 0.06$ & $33 \pm 8$ \\
2839 & $1: 11: 45.94$ & $-35: 02: 04.2$ & $26.95_{-0.23}^{+0.27}$ & $28.38_{-0.32}^{+0.44}$ & $>28.48$ & $0.43 \pm 0.10$ & $0.20 \pm 0.04$ & $32 \pm 12$ \\
90 & $1: 11: 53.63$ & $-35: 06: 26.9$ & $26.74_{-0.2}^{+0.23}$ & $28.16_{-0.21}^{+0.26}$ & $>28.68$ & $0.52 \pm 0.10$ & $0.24 \pm 0.05$ & $31 \pm 8$ \\
1680 & $1: 11: 55.75$ & $-35: 03: 36.2$ & $26.08_{-0.25}^{+0.31}$ & $27.46_{-0.25}^{+0.32}$ & $27.25+0.25$ & $0.96 \pm 0.24$ & $0.45 \pm 0.11$ & $29 \pm 10$ \\
100 & $1: 11: 28.09$ & $-35: 06: 25.9$ & $26.45_{-0.20}^{+0.23}$ & $27.83_{-0.28}^{+0.37}$ & $>27.37$ & $0.68 \pm 0.13$ & $0.32 \pm 0.06$ & $29 \pm 10$ \\
1572 & $1: 11: 47.06$ & $-35: 03: 23.6$ & $25.72_{-0.11}^{+0.12}$ & $27.02_{-0.14}^{+0.16}$ & $27.64_{-0.29}^{+0.37}$ & $1.34 \pm 0.14$ & $0.62 \pm 0.07$ & $27 \pm 4$ \\
2478 & $1: 11: 37.99$ & $-35: 01: 13.5$ & $25.51_{-0.09}^{+0.10}$ & $26.81_{-0.11}^{+0.11}$ & $27.7_{-0.28}^{+0.36}$ & $1.63 \pm 0.14$ & $0.76 \pm 0.07$ & $26 \pm 3$ \\
2809 & $1: 11: 33.74$ & $-35: 02: 00.2$ & $25.33_{-0.20}^{+0.23}$ & $26.56_{-0.16}^{+0.18}$ & $27.47_{-0.27}^{+0.34}$ & $1.92 \pm 0.37$ & $0.89 \pm 0.17$ & $24 \pm 5$ \\
\hline
\end{tabular}

\section{APPENDIX B: RADIATIVE TRANSFER SIMULATIONS}

In this section, we briefly describe the hydrodynamical and Radiative Transfer (RT) simulations used throughout the paper. A detailed description will be provided in a separate paper (Cantalupo \& Haehnelt, in preparation).

We have performed cosmological Adaptive Mesh Refinement (AMR), hydrodynamical simulations using the publicly available code Ramses, version 3.03 (Teyssier 2002). The computational domain is composed by a series of four nested boxes at increasing, root-grid resolution. The largest box has a linear size of 40 comoving Mpc, corresponding roughly to the FWHM of the NB filter used in our FORS2 observation at $z=2.4$, while the high-resolution box has a linear size of 10 comoving $\mathrm{Mpc}$, corresponding roughly to the FOV of our FORS2 observation at $z=2.4$. The equivalent base-grid resolution in the high-resolution region corresponds to a $\left(1024^{3}\right)$ grid with a dark-matter particle mass of about $1.8 \times 10^{6} M_{\odot}$. We allowed 6 additional refinements level, reaching a maximum spatial resolution of about 0.6 comoving kpc, i.e. about 180 proper pc at $z=2.4$. The box has been centred on the most massive halo at $z=2.4, M_{\mathrm{DM}} \sim 10^{13} M_{\odot}$, as a representative host of a luminous quasar. We include photo-ionization from the cosmic UV background (Haardt \& Madau 2012) using a self-shielding above a critical density as derived by Schaye (2001). In each cell, gas is converted into star particles following a Schmidt law with a density threshold of 1 atom $\mathrm{cm}^{-3}$ and a star formation timescale of 3 Gyr (see, e.g. Dubois \& Teyssier 2008). We include metal cooling, metal enrichment and supernova feedback as described in Dubois \& Teyssier 2008.

To produce the "quasar-on" models, the results of the hydrodynamical simulation have been post-processed with our RT code RADAMESH (Cantalupo \& Porciani 2011) to include the time-dependent ionization effects due a bright quasar with Lyman Limit luminosity of $L_{11}=5 \times 10^{31} \mathrm{erg} \mathrm{s}^{-1}$ and spectral slope of $\alpha=-1.7$ (Telfer et al. 2002). We include hydrogen and helium ionization and we evolved the temperature of the gas consistently with the ionization state using a large number of frequency bins (50) for the ionizing radiation. We assumed a quasar age of 20Myr, large enough to illuminate the whole simulation box.

In order to obtain simulated Ly $\alpha$ images we post-processed the "quasar-off" and "quasar-on" runs with our threedimensional Ly $\alpha$ Monte Carlo RT (Cantalupo et al. 2005) including the gas velocity field and inhomogeneous temperature distribution. As a source of Ly $\alpha$ radiation we included HII recombination and internal star formation, while we have decided to exclude contribution from HI collisional excitations. This is due to the fact that a proper modeling of this emission for dense systems would have required the coupling of hydro and RT, given its strong temperature dependence for a photo-ionized gas (e.g., Cantalupo et al. 2008; see also, e.g., Furlanetto et al. 2005, Faucher-Giguère et al. 2010 and Rosdahl \& Blaizot 2011 for further discussion), currently beyond the capabilities of the large majority of numerical studies. We note however that the contribution from HI collisional excitations is likely negligible if the gas is highly ionized, as expected in proximity of an ultraluminous quasar. Partially ionized systems, e.g., optically thick clouds to the quasar radiation could present instead significant contribution from HI collisional excitations. For these sources, the simulated Ly $\alpha$ emission, in absence of dust, needs to be considered a conservative estimate.

We computed the Ly $\alpha$ emissivity from the simulated Star Formation Rate (SFR) converting the H $\alpha$-SFR relation of Kennicutt (1998) to a Ly $\alpha$-SFR relation using the Case B line ratio (e.g., Osterbrock 1989 ), obtaining: $\mathrm{SFR}=9.1 \times 10^{-43} L_{\mathrm{ly} \alpha}$ $\mathrm{erg} \mathrm{s}^{-1}$. We do not include dust absorption, likely important only for the most massive Ly $\alpha$ emitters powered by internal starformation, while the "typical" Ly $\alpha$ emitter seems to contain very little dust (e.g., Guaita et 2011). Nevertheless, continuumselected samples of star forming galaxies at $z \sim 2.5$ (e.g., Steidel et al.2011) show that only about half of the galaxies present Ly $\alpha$ in emission and only $20 \%$ of them have $\mathrm{EW}_{0}>20 \AA$, the standard selection cut. In other words, not every star-forming galaxy is a $\operatorname{Ly} \alpha$ emitter and we must take this into account when deriving the simulated Luminosity Functions. In particular, when we computed the simulated Luminosity Function in the "quasar-off" model (where Ly $\alpha$ is only powered by star formation), we have used a normalization factor $n_{\mathrm{ly} \alpha}=0.5 / 3.7=0.13$, where the factor 3.7 is necessary to renormalize for the overdensity of the simulated high-resolution region. Note that, given the large uncertainties related to the Ly $\alpha$ fraction and its evolution with galaxy luminosity (e.g., Stark et al. 2010), $n_{\mathrm{ly} \alpha}$ is essentially a free parameter in our model. It is remarkably, however, that with the adopted value we are able to obtain a simulated LF very similar to the observed LF by Grove et al. (2009) and Hayes et al. (2010). In the "quasar-on" simulation we use the same rescaling factor, since we are interested in the 
change of slope rather than normalization. Also in this case, however, the adopted value of $n_{\mathrm{ly} \alpha}$ - combined with the steepening of the LF - seems to produce the right amount of sources, regardless of the possible difference in overdensity between quasar and random fields. Note that in the "quasar-on" simulation we have assumed isotropic quasar emission, while in reality only a fraction of the volume may be illuminated by the quasar. This parameter would be degenerate with field overdensity. In other words, the adopted value of $n_{\mathrm{ly} \alpha}$ for the fluorescent sources may also be consistent with a cosmic volume 4 times more dense than a random field, if the quasar is only "illuminating" one fourth of such volume. 